\title{
Las cuentas medioambientales físicas en el INE: situación actual y perspectivas futuras
}

\section{Physical Environmental Accounts of the Spanish Statistical Office: Current and Future Prospects}

\author{
Fernando Celestino \\ Julia Maestro \\ Luis Martín \\ Instituto Nacional de Estadística
}

\begin{abstract}
Resumen
Las cuentas medioambientales físicas muestran, en términos físicos, como el medioambiente contribuye al desarrollo económico (por ejemplo, por medio de la extracción de recursos naturales), los impactos que la economía tiene sobre el medioambiente (por ejemplo, consumo de energía, emisiones a la atmósfera, generación de residuos, contaminación, pérdida de biodiversidad), así como el agotamiento del capital natural (recursos energéticos naturales, agua, bosques y tierra). En este artículo se presentan las cuatro cuentas medioambientales físicas que el INE lleva a cabo en la actualidad (materiales físicos, flujos físicos de la energía, emisiones a la atmósfera y residuos) por mandato de los respectivos reglamentos comunitarios y en aplicación de las normas metodológicas emanadas de la Oficina de Estadística de la Unión Europea (Eurostat). De cada una de ellas, se presenta el marco conceptual, la metodología y estructura, el proceso de elaboración, los principales resultados y, finalmente, los indicadores que pueden derivarse de las cuentas.
\end{abstract}

Palabras clave: medioambiente, flujos físicos, residuos energéticos, residuo, emisiones, ajustes de residencia.

Clasificación JEL: Q56.

\begin{abstract}
Physical Environmental Accounts show in physical terms, how the environment contributes to economic development (for example, through the extraction of raw materials), the impacts that the economy has on the environment (for example, energy consumption, air emissions, generation of waste, pollution and loss of biodiversity), as well as the depletion of the natural capital (energy natural materials, water, forests and land). This article presents the four physical environmental accounts currently carried out by INE (physical materials, physical energy flows, air emissions and waste) as mandated by the respective Community regulation and following the methodological standards issued by Statistical Office of the European Union (Eurostat). For each of these accounts, the article describes the conceptual framework, the methodology, the development process, the main results and finally, the indicators that can come from them.
\end{abstract}

Keywords: environment, physical flows, energy residuals, waste, emissions, resident adjustments. 


\section{Introducción}

Las cuentas medioambientales físicas tienen por objeto el estudio de las interrelaciones entre el sistema económico y el medioambiente, cuantificando en magnitudes físicas el impacto y en su caso deterioro, que sufre el medio natural por efecto de la actividad humana y económica (consumo de recursos naturales, extinción de la biodiversidad, contaminación, calentamiento de la atmósfera, etc.). Por tanto, al proporcionar dichas cuentas información en cantidades físicas, es posible llevar a cabo estudios y balances de recursos naturales que permiten identificar las variables y los parámetros que conforman las citadas interrelaciones.

Conceptualmente, las presiones que sufre el medioambiente por la actividad económica pueden considerarse como el «efecto acción» de esta problemática, siendo el de «reacción» las medidas que el sistema económico, las autoridades administrativas y la propia sociedad toman para anular o paliar estos efectos (gastos en protección medioambiental, subvenciones e impuestos medioambientales, etc.), medidas que tienen su concreción en las cuentas medioambientales monetarias.

Seguidamente, se presentarán las cuatro cuentas ambientales físicas que actualmente lleva a cabo por mandato de Eurostat el Instituto Nacional de Estadística (INE). Esta relación no es exhaustiva ya que existen otras cuentas que bien están a cargo de otros organismos (como por ejemplo la Cuenta de los Bosques), que bien no tienen un reglamento de la UE que obligue a su realización (Cuentas del Agua) o que bien se encuentran aún en un estadio experimental (como podrían ser la Cuenta de los Ecosistemas -biodiversidad-) y la Cuenta del Capital Natural.

La periodicidad de las cuentas que aquí se presentan es anual y el plazo de transmisión a Eurostat, establecido por el pertinente Reglamento, es de 24 meses después del año de referencia de los datos. Así, los datos de referencia temporal 2018 se han transmitido a dicho organismo y se publican en la web del INE a lo largo del tercer y cuarto trimestre de 2020.

Estas cuatro cuentas físicas medioambientales tienen los mismos límites conceptuales que el Sistema Europeo de Cuentas Económicas (SEC), el cual se basa en el denominado «principio de residencia». Conforme se establece en el SEC ${ }^{1}$, una unidad económica es residente en un país cuando tiene un centro de interés económico en el territorio geográfico de ese país, es decir, cuando se dedica por un periodo prolongado (un año o más) a realizar actividades económicas en ese territorio, independientemente de su nacionalidad, de su forma jurídica o de su presencia física. Así, las cuentas registran las actividades de las unidades económicas residentes en el país objeto de estudio, con independencia de la localización geográfica dónde se generan estos flujos.

En este contexto metodológico es preciso señalar que las estadísticas de energía, así como los inventarios nacionales de emisiones se basan en el «principio de territorialidad», es decir, que miden las actividades que se llevan a cabo dentro de los

\footnotetext{
${ }^{1}$ https://unstats.un.org/unsd/nationalaccount/docs/SNA2008Spanish.pdf
} 
límites geográficos y administrativos de un país, tanto por las unidades residentes como no residentes. Por el contrario, las cuentas se basan en el principio de residencia anteriormente definido que es la base para el estudio de la actividad económica de un país.

\section{Cuenta de Flujos de Materiales}

\subsection{Marco conceptual}

Durante las últimas décadas se ha reforzado de forma drástica el foco social y mediático sobre el uso sostenible de los recursos naturales. En este sentido, en «Nuestro futuro común» (Brundtland, 1987), se define el desarrollo sostenible como la satisfacción de «las necesidades de la generación presente sin comprometer la capacidad de las generaciones futuras para satisfacer sus propias necesidades». Este movimiento ha ido ganando inercia durante los últimos años con el Acuerdo de París sobre el cambio climático y la Agenda 2030 de la ONU para el Desarrollo Sostenible en 2015. En especial, dentro de la Unión Europea cabe destacar el Pacto Verde Europeo que establece el ambicioso objetivo de convertir Europa en el primer continente climáticamente neutral en 2050.

Así, se trata de lograr, de manera equilibrada, los siguientes objetivos: desarrollo económico, desarrollo social, la protección del medioambiente y la conservación del capital natural. Conseguir ese equilibrio implica necesariamente racionalizar el uso de los recursos naturales.

Normalmente, encontramos asociado al crecimiento de la actividad económica, un incremento de la necesidad de diversos tipos de materiales y de recursos energéticos, y a menudo se necesita importar de otros países gran parte de los mismos. Cabe destacar en este aspecto que en España en 2019 se consumieron 356,9 toneladas de materiales por cada millón de euros de PIB y que las importaciones supusieron un 42,5\% de esos inputs de materiales (Instituto Nacional de Estadística [INE], 2020). Racionalizando el uso de los recursos naturales, se otorga un mayor valor económico a cada unidad empleada. De esta manera, uno de los factores fundamentales a monitorizar, es si la utilización de los recursos naturales directamente extraídos del medioambiente tiende o no a decrecer, ya que con ello obtendremos información que nos permita evaluar las presiones sobre los recursos, como parte del conjunto de información necesario para medir el desacoplamiento del crecimiento económico y el medioambiente (European Commission, 2019).

El primer paso para poder tomar medidas encaminadas a disminuir la intensidad en el uso de los recursos, es contar con unas fuentes de información fiables. Resulta por tanto crucial medir los siguientes aspectos:

- Recursos naturales de los que dependen nuestra producción y nuestro consumo, midiendo tanto la cantidad como la naturaleza de los mismos. 
- Cuántos y cuáles de esos recursos extraemos de nuestro propio medio natural y cuántos y cuáles son importados.

- Qué recursos perdemos en forma de desechos o cuáles abandonan nuestra economía mediante las exportaciones.

La Cuenta de Flujo de Materiales (en adelante CFM) se elabora para cubrir esos objetivos y muestra los inputs físicos de materiales que entran en el sistema económico nacional y los outputs que salen a otras economías o al medio natural en unidades físicas. Los principales aspectos de la CFM vienen recogidos en el Reglamento (UE) 691/2011 del Parlamento Europeo y del Consejo de 6 de julio de 2011 relativo a las cuentas económicas medioambientales. Entre otros aspectos se recoge que la CFM se elaborará en términos físicos (toneladas) y se transmitirá anualmente. El plazo de transmisión establecido por el Reglamento es de 24 meses después del año de referencia de los datos, aunque en la práctica se elaboran indicadores avanzados para las principales categorías 12 meses después del último año de referencia de los datos.

Con esto conseguimos obtener un conjunto de indicadores agregados del uso de los recursos naturales. Como veremos más adelante, relacionando los resultados de estas mediciones con datos monetarios en términos de PIB y con otros datos económicos y de empleo, se pueden derivar indicadores de productividad o de eficiencia en el uso de los recursos naturales, e indicadores de intensidad (o necesidad de materiales), y analizar así las tendencias de todos ellos. También, se pueden utilizar para calcular otros indicadores como el consumo de materiales per cápita, considerando el tamaño de la población, u otros indicadores demográficos (INE, 2020).

\subsection{Metodología y estructura}

Esta cuenta nos ofrece información sobre los inputs físicos de materiales que entran en el sistema económico nacional (extracción nacional e importaciones) y los outputs a otras economías (exportaciones) o al medio natural, clasificados en función de su naturaleza.

La distinción entre stocks y flujos constituye un principio fundamental de un sistema de flujos de materiales. En general, un flujo es una variable que mide una cantidad en un periodo de tiempo, mientras que un stock es una variable que mide una cantidad en un momento dado. Las CFM miden los flujos de entradas, salidas y las variaciones de stocks de materiales en la economía, en unidades de masa por año.

Las CFM son coherentes con el Sistema Europeo de Cuentas (SEC), y al igual que las Cuentas Nacionales, siguen el principio de residencia.

En estas cuentas son relevantes dos tipos de flujos de materiales que cruzan los límites del sistema:

1. Flujos de materiales entre la economía nacional y su medioambiente natural. Consisten en la extracción de materiales (en bruto, crudo o virgen) del 
medioambiente natural y en el vertido de otros (llamados a menudo «residuos») y emisiones.

2. Flujos de materiales entre la economía nacional y la economía del resto del mundo. Comprenden las importaciones y exportaciones.

Todos los flujos que cruzan estos límites del sistema se incluyen en las CFM, así como las acumulaciones de stocks producidos. Los denominados flujos ocultos o extracción nacional no usada (materiales que se movilizan de forma intencionada y por medios tecnológicos pero que no se consideran apropiados o no están destinados a ser utilizados en la economía) no cruzan esas fronteras y, por tanto, no se representan en las CFM. La economía nacional se trata globalmente en las CFM, y no se describen las entregas de productos entre ramas de actividad. Asimismo, se excluyen los flujos naturales en el interior del medioambiente natural. (INE, 2002) (Gráfico 1).

La extracción nacional (usada) comprende la cantidad anual de materiales sólidos, líquidos y gaseosos (sin incluir el aire y el agua) extraídos del medio natural para ser usados como inputs en la economía. Las variables que describen la extracción nacional se clasifican en función del tipo de material que se extrae, de acuerdo a la clasificación de materiales establecida por el Anexo III del Reglamento y se agrupan en las siguientes categorías principales:

1. Biomasa. Engloba toda la biomasa de origen vegetal extraída y la consumida directamente por la cabaña ganadera, así como la captura de peces y la caza de animales. La biomasa constituida por la cabaña ganadera y sus productos derivados se consideran productos secundarios y por tanto no se contabilizan.

\section{GRÁFICO 1}

\section{ESQUEMA GENERAL DEL BALANCE DE MATERIALES}

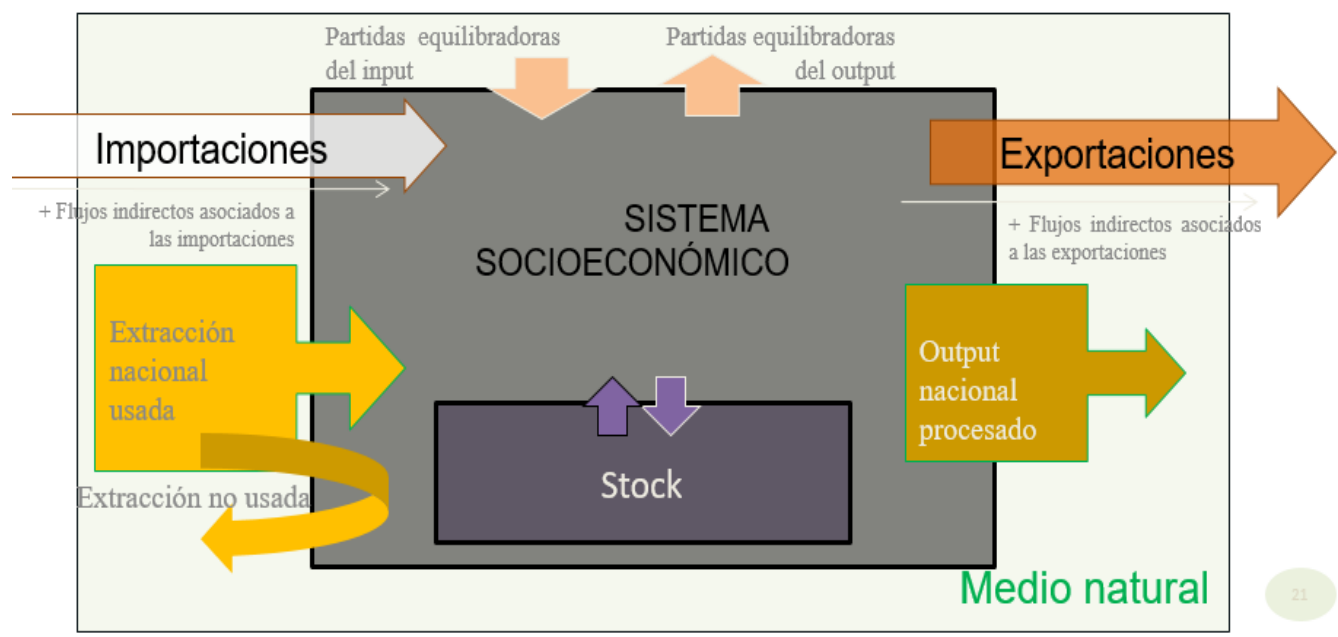

Fuente: Elaboración propia. 
2. Minerales metálicos. En este tipo de minerales se considera pro memoria el contenido neto en minerales metálicos, pero se contabiliza todo el material extraído que contiene el metal (mineral en bruto).

3. Minerales no metálicos. En el caso de los minerales no metálicos se supone que las diferencias entre el mineral en bruto y la producción mineral reportada generalmente no son relevantes.

4. Combustibles fósiles. Incluye carbones y otros recursos sólidos, así como recursos líquidos y gaseosos como el petróleo y el gas natural.

Las importaciones y exportaciones físicas comprenden todas las mercancías importadas o exportadas, contabilizadas en unidades de masa según el peso que tienen en el momento de cruzar las fronteras. Al contrario de lo que ocurre en la extracción nacional, donde los recursos son siempre materias primas, las mercancías intercambiadas en el comercio exterior incluyen bienes en todas sus etapas de transformación, desde productos básicos a bienes acabados y se clasifican, de forma coherente con la clasificación de la extracción nacional, en cerca de 60 categorías descritas en el Anexo III del Reglamento, atendiendo a la materia principal que los compone. La inclusión en uno u otro agregado se lleva a cabo según el nivel más detallado ( 8 dígitos) del sistema de codificación INTRASTAT CN8 o nomenclatura combinada (European Commission, 2020), que es la clasificación usada en la Unión Europea para recoger y procesar datos de comercio exterior.

En virtud del principio de residencia, tanto importaciones como exportaciones incluyen una partida de ajuste por el combustible proporcionado para el transporte internacional: en el exterior el comprado por unidades residentes (importaciones), y en el interior el comprado por unidades no residentes (exportaciones).

Las principales agrupaciones en importaciones y exportaciones son:

1. Biomasa y productos de biomasa.

2. Minerales metálicos (materias primas y productos terminados).

3. Minerales no metálicos (materias primas y productos terminados).

4. Combustibles fósiles.

5. Otros productos (demasiado complejos en su composición para poder ser asignados a otras categorías).

6. Residuos (importados o exportados) para su tratamiento o su eliminación final.

El output nacional procesado a la naturaleza se define como la cantidad total de materiales liberados al medioambiente después de haber sido utilizados en la economía nacional. Estos flujos tienen lugar durante el procesamiento, fabricación, uso y etapas de eliminación final de la cadena de producción y consumo y se clasifican en emisiones a la atmósfera, residuos depositados en vertederos, vertidos a las aguas, disipación de productos y pérdidas disipadas de materiales. El reglamento actual no obliga a la recopilación de estos datos, puesto que gran parte de esta información se 
puede encontrar de forma mucho más detallada en otras cuentas ambientales, pero las partidas correspondientes se recogen para su envío de forma voluntaria y pronto serán incorporadas a la publicación anual del INE.

Completando el sistema de cuentas tendríamos las partidas equilibradoras del input y el output (estimaciones de los flujos de agua y aire que tienen lugar en determinados procesos y que afectan significativamente al balance de masas, como pueden ser la demanda de oxígeno o las emisiones de $\mathrm{CO}_{2}$ y de vapor de agua en los procesos de combustión y en la respiración humana y del ganado, requerimientos de agua para la producción de bebidas exportadas, agua procedente de la deshidratación de productos de biomasa, etc.). Estas partidas se reportan en tablas específicas y no se incluyen en los indicadores agregados ni son requerimiento del Reglamento que regula esta cuenta; sin embargo, es precisa una buena estimación de estas partidas cuando se calculan las acumulaciones netas de stocks como diferencia entre el total de inputs y el total de outputs en el balance de flujos de materiales.

La clave a la hora de entender la CFM es el manual de Eurostat (Eurostat, 2018) que presenta los fundamentos teóricos y conceptuales de la cuenta y aporta guías de ayuda para la correcta recopilación de los datos requeridos por el reglamento. Cabe destacar que este manual se ha revisado recientemente para adaptar los conceptos a los cambios efectuados en los sistemas de Contabilidad Nacional y para relacionar cada dimensión estudiada en la cuenta con los indicadores derivados correspondientes.

\subsection{Elaboración}

Al tratarse de estadísticas de síntesis, las CFM combinan y concilian, anualmente, datos procedentes de diversas fuentes estadísticas, siendo las principales para esta operación las Cuentas Nacionales y otras cuentas ambientales elaboradas por el INE, las Estadísticas de Comercio Exterior de la Agencia Tributaria, las Estadísticas mineras y energéticas del Ministerio para la Transición Ecológica y las Estadísticas Agrarias, Forestales y Pesqueras del Ministerio de Agricultura, Pesca y Alimentación.

Una vez recopilada la información referente a los flujos de entrada y salida del sistema, considerando como recursos aquellos flujos que añaden alguna cantidad de materiales a la economía y como empleos los flujos que reducen la cantidad de materiales, esta cuenta permite la obtención de distintos indicadores. La elección de uno u otro dependerá del enfoque político y de la utilidad y aplicabilidad probada para cada indicador, siendo los fundamentales los siguientes:

- Registrando, como total de recursos disponibles, la entrada directa de materiales en el sistema económico procedentes del medio natural nacional y del resto del mundo, es decir, extracción nacional e importaciones, obtenemos el input directo de materiales (IDM). 
- Cuando detraemos del total de recursos disponibles los empleados en las exportaciones, obtenemos el consumo nacional de materiales (CNM), que mide la cantidad total de materiales usados directamente en la economía.

- El balance comercial físico mide el excedente o déficit del comercio físico de la economía, es decir las importaciones menos las exportaciones. Este indicador se utiliza como aproximación a la dependencia de recursos del exterior.

- Se estima que en 2017 un tercio de los 92 billones de toneladas de materiales extraídos en la economía global fueron empleados para producir los productos destinados a la exportación (UNEP, 2020). Si calculamos las importaciones y las exportaciones en términos de materias primas equivalentes (teniendo en cuenta los flujos indirectos), podremos calcular también el indicador de consumo nacional de materias primas (RMC, por sus siglas en inglés), que muestra el total de materias primas extraídas del medioambiente que son necesarias para satisfacer la demanda de un país, independientemente del lugar del mundo donde tuvo lugar la extracción de esas materias primas.

Como referencia, en el Gráfico 2, se pueden ver los datos de la economía española en el año 2019. Así, mediante la suma de la extracción nacional y las importaciones, obtenemos el dato del IDM. La parte central del Gráfico 2 muestra la desagregación del IDM por tipo de recurso. En la parte derecha del Gráfico 2 podemos calcular, por diferencia con los materiales exportados, el CNM. En el avance del 2019, los datos reflejan que el consumo nacional de materiales alcanzó los 426,1 millones de toneladas, lo que supone una disminución del $3 \%$ respecto del año anterior.

Los últimos indicadores que se calculan con esta información son los denominados indicadores relativos. Estos nos permiten entender el funcionamiento de la eco-

\section{GRÁFICO 2}

BALANCE DEL CONSUMO NACIONAL DE MATERIALES (2019)

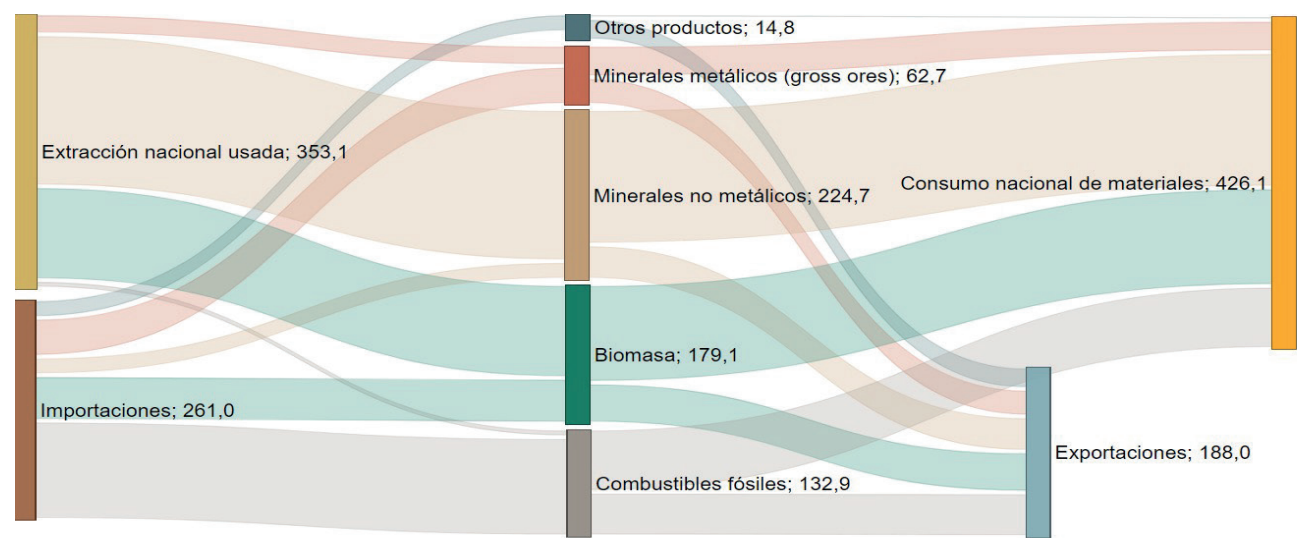

NOTA: Unidades: millones de toneladas.

FUENTE: Elaboración propia. 
nomía desde una visión holística, es decir, analizando las interacciones entre factores económicos, materiales y humanos. Así, mediante la relación de los indicadores físicos anteriormente mencionados con los monetarios, se permite:

- Calcular indicadores de productividad (eficiencia) de los recursos, siendo el más utilizado, la ratio entre el PIB producido y el consumo nacional de materiales.

- Calcular indicadores de intensidad en el uso de los recursos naturales. Para este fin suele emplearse el inverso de la productividad, es decir la ratio entre el consumo nacional de materiales y el PIB o la ratio entre IDM/PIB.

- Analizar las tendencias y patrones de evolución.

En ocasiones se relacionan también los indicadores absolutos con el número de habitantes, siendo el más relevante el CNM per cápita (toneladas de materiales consumidas por habitante).

Uno de los mayores retos que afrontaremos durante las próximas décadas es el establecimiento de la denominada economía circular. Este modelo se basa en el mantenimiento de los recursos materiales dentro del proceso productivo y consuntivo durante el mayor plazo posible, a la vez que se minimiza la generación de residuos (Eurostat, 2020). Este cambio de paradigma plantea también nuevos retos desde el punto de vista de desarrollo de indicadores para ofrecer datos sobre:

- El flujo de determinados recursos por tipo de material y uso final de los mismos.

- Importación y exportación en materias primas equivalentes.

- Importación y exportación en materias primas equivalentes por uso final de los productos.

- Tasas de uso de materiales secundarios en relación con la cantidad total utilizada en la economía.

- Tasas de uso de materiales secundarios desagregada por tipo de material.

\subsection{Situación actual. Principales indicadores ${ }^{2}$}

Los primeros estudios piloto de la CFM en España recogen la serie contable 1996-2000. Se realizaron en el año 2002, a fin de preparar el Sistema Estadístico español a los futuros requerimientos del Sistema Estadístico Europeo. Posteriormente se continuó con las estimaciones en las bases 2008 y 2010 de forma paralela a las sucesivas estimaciones de las Cuentas Nacionales. Las últimas estimaciones de la CFM, acordes al Reglamento y base de Cuentas Nacionales actuales, corresponden a la serie 2008-2019.

Los gráficos mostrados a continuación pretenden dar una visión actual de los indicadores que se pueden obtener a partir de esta cuenta.

\footnotetext{
2 Este apartado está basado en la nota de prensa del Instituto Nacional de Estadística «Cuentas medioambientales: Cuenta de flujos de materiales. Datos avance 2019» (2020, 16 de diciembre). 
Observando la evolución del total de materiales extraídos del medio natural, desagregado por sus componentes principales (Gráfico 3), se puede apreciar la fuerte reducción de la extracción de toneladas de recursos naturales en los primeros años de la serie. En particular, muestra un desplome de la obtención de minerales no metálicos ligada al fuerte descenso de la actividad de la construcción.

\section{GRÁFICO 3}

EVOLUCIÓN DE LA EXTRACCIÓN NACIONAL DE MATERIALES

(SERIE 2008-2019)

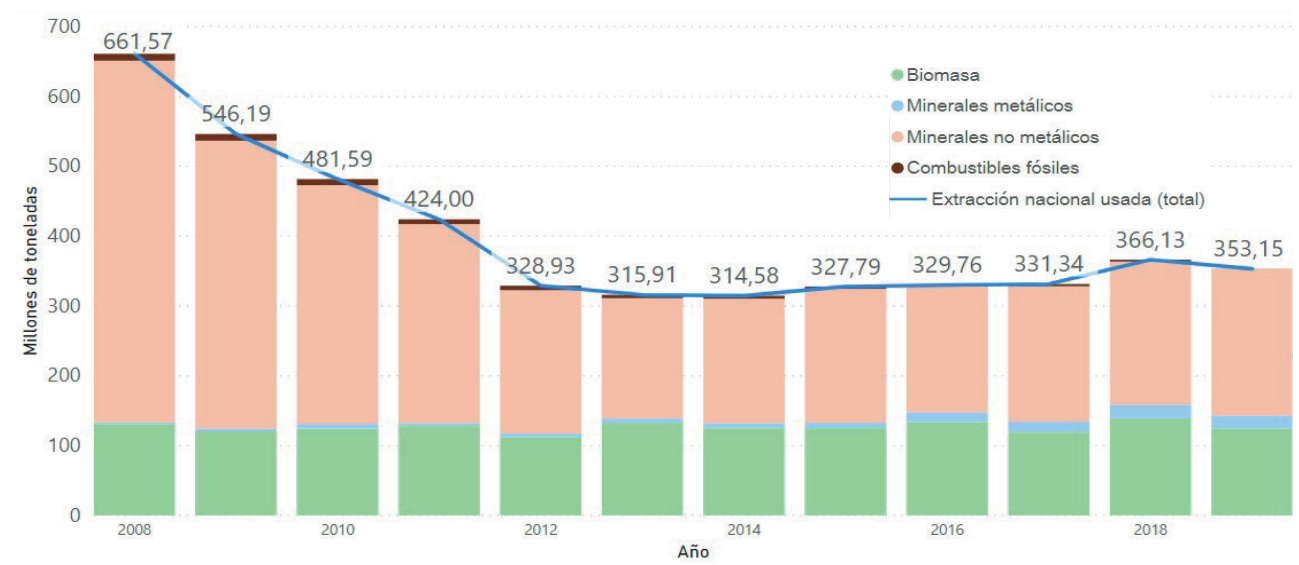

FUENTE: Elaboración propia.

Un análisis más detallado de los componentes de la extracción nacional usada, en particular de los minerales no metálicos (Gráfico 4), nos permite apreciar claramente la preponderancia de los materiales relacionados con la construcción con respecto al total de materiales extraídos.

\section{GRÁFICO 4 \\ COMPONENTES DE LA EXTRACCIÓN DE MATERIALES EN ESPAÑA (2019)}

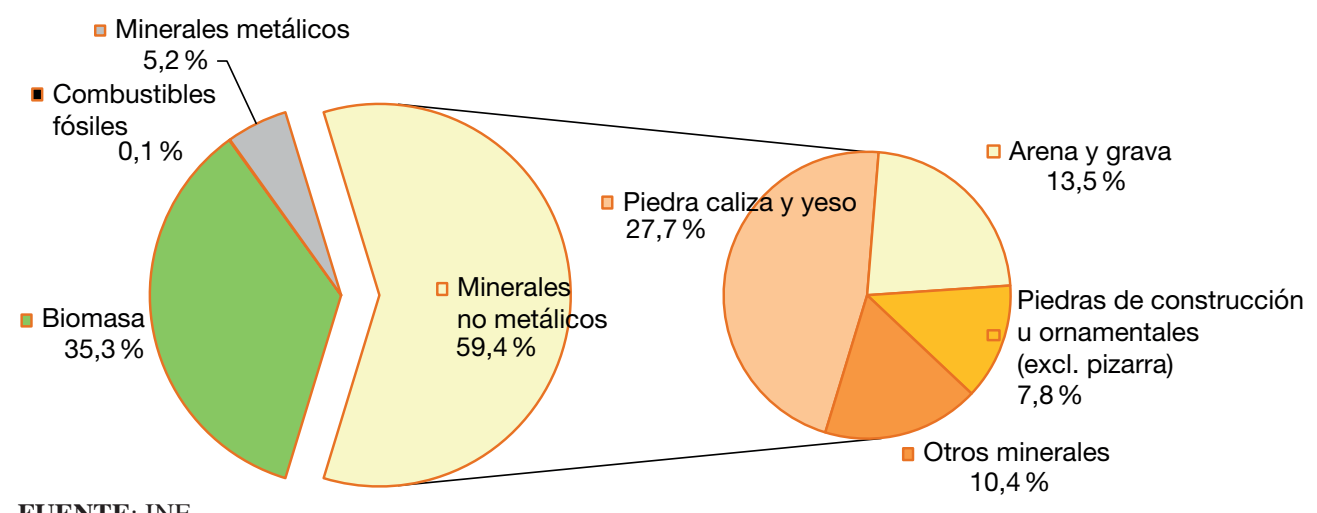

FUENTE: INE. 
El balance comercial físico en el avance del año 2019 (INE, 2020) pone en relevancia, al igual que en años anteriores, la gran dependencia exterior en lo que a combustibles fósiles se refiere (carbón, crudos del petróleo, gas natural y derivados). Por el contrario, España en 2019 fue exportadora neta de minerales no metálicos, una tendencia al alza en los últimos años de la serie.

\section{GRÁFICO 5 \\ CONSUMO NACIONAL DE MATERIALES POR HABITANTE, PRODUCTO INTERIOR BRUTO Y PRODUCTIVIDAD DEL CONSUMO DE MATERIALES}

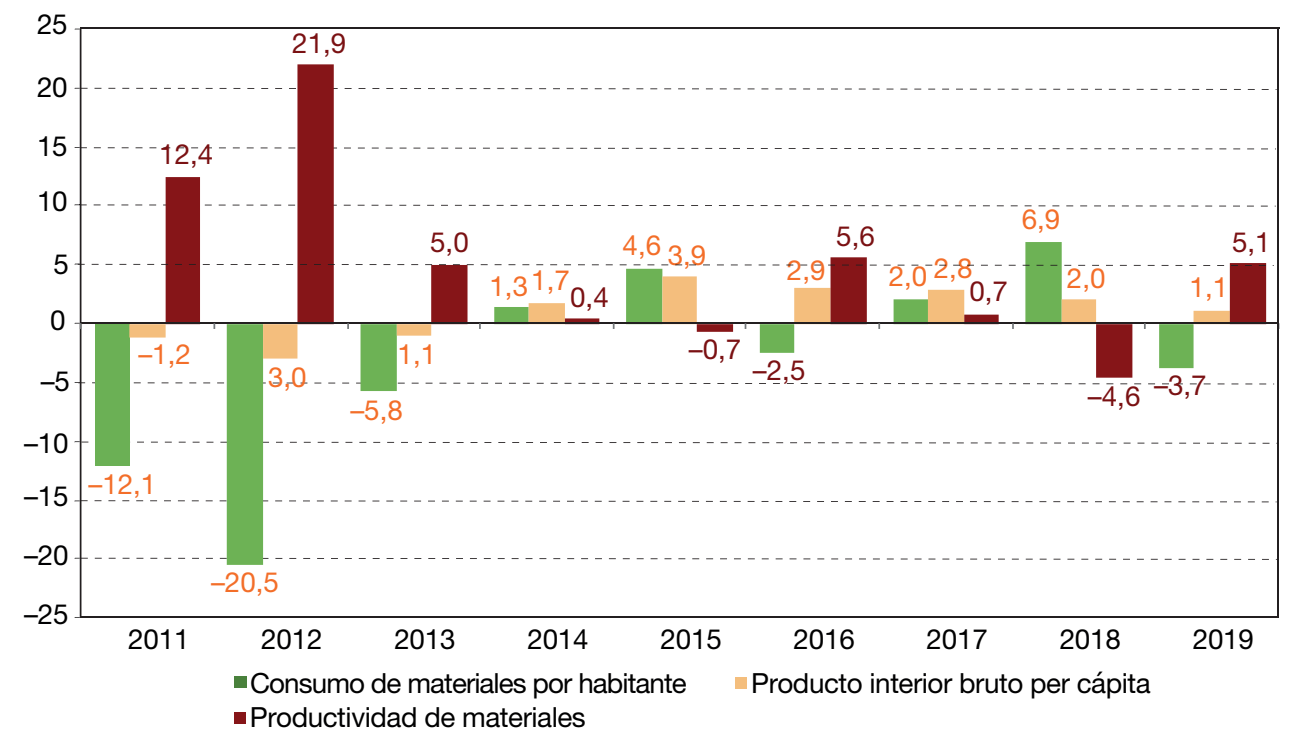

NOTA: Unidad: Tasas de variación anuales.

FUENTE: Elaboración propia.

Los datos de la CFM nos permiten también analizar las tendencias de los principales indicadores de consumo y productividad de materiales (Gráfico 5). En términos per cápita, el consumo de materiales por habitante alcanzó las 9 toneladas, lo que supone un 3,7\% menos que en el periodo anterior. Los datos de productividad, medida en cantidad de producto interior bruto (PIB) por tonelada de materiales consumida, indican un incremento del $5,1 \%$ respecto al año anterior, situándose en $2.801,8$ euros por tonelada. 
Se puede ver que en 2016 se dio un aumento del crecimiento económico no vinculado al aumento del consumo de materiales. En otras ocasiones como en 2014 o en 2017 el incremento en la tasa interanual del PIB llevó aparejado un incremento incluso mayor en la tasa interanual del consumo de materiales por habitante, lo que repercutió negativamente en el dato de la productividad. El análisis de estas tendencias en el tiempo puede aportar también información sobre el desacoplamiento del uso de materiales y el crecimiento económico.

Los datos actuales de la CFM junto con el resto de cuentas físicas elaboradas por el INE, permiten estimar el balance completo de materiales, únicamente para las toneladas totales (Gráfico 6). El reto futuro de estas cuentas es, por tanto, poder estimar la mayor cantidad posible de estos flujos por tipo de recurso, con una especial atención a los considerados recursos críticos.

\section{GRÁFICO 6 \\ DIAGRAMA DE FLUJO DE MATERIALES ESPAÑA (2018)}

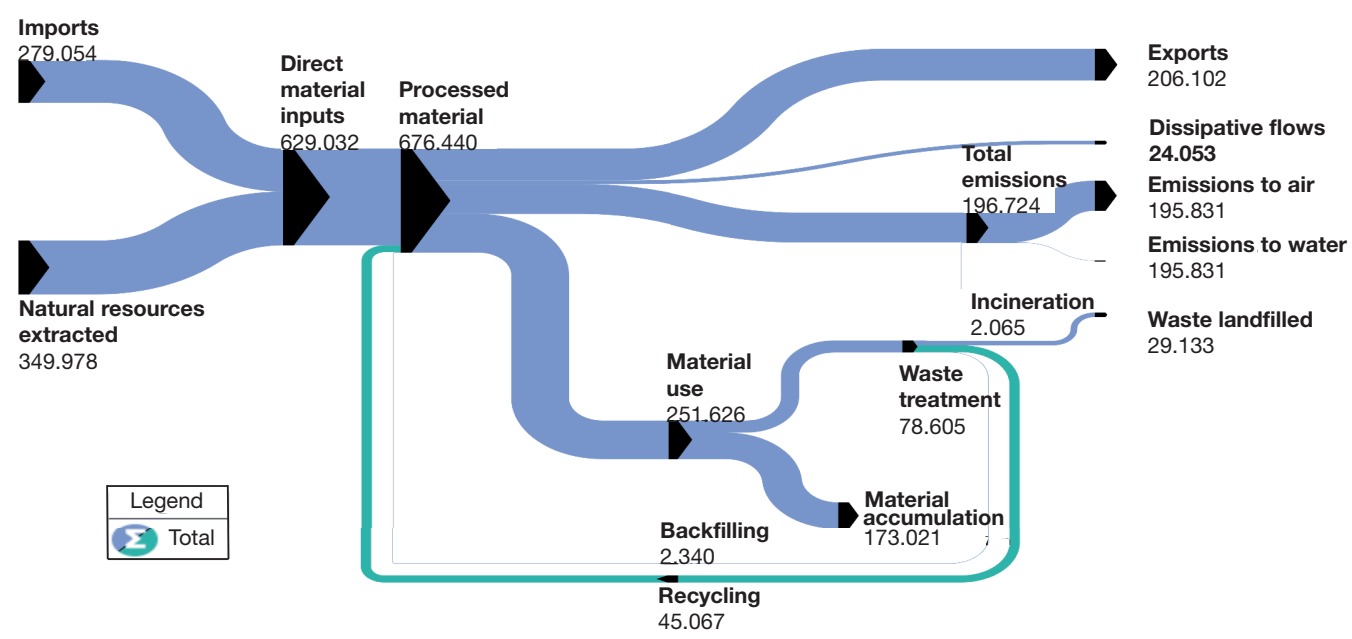

FUENTE: Eurostat.

\section{Cuenta de Flujos Físicos de la Energía ${ }^{3}$}

\subsection{Marco conceptual}

La preocupación sobre la sostenibilidad de la extracción de los recursos naturales de carácter energético, llevó a que los indicadores sobre el uso de esos recursos formaran parte de los diecisiete objetivos de la Agenda 2030 para el Desarrollo

3 Este epígrafe está basado en el artículo «Las cuentas medioambientales y la cuenta de los flujos físicos de la energía», Energía Industrial, 408 (Celestino Rey et al., 2019). 
Sostenible, aprobada por la Asamblea General de Naciones Unidas en septiembre de 2015. Por otra parte, la Cuenta de flujos físicos de la energía ha venido a complementar la Cuenta de emisiones a la atmósfera que venía llevándose a cabo desde los inicios de la década de 2000, en tanto en cuanto más del $90 \%$ de dichas emisiones son producidas por el uso de combustibles fósiles

El INE lleva a cabo con periodicidad anual y desde el año 2014 (referencia temporal de los datos) la Cuenta de flujos físicos de la energía (CFFE), mandatado por el Reglamento (UE) 538/2014 del Parlamento Europeo y del Consejo, que incorporó tres nuevas cuentas medioambientales que vinieron a sumarse a las que ya había establecido el Reglamento (UE) 691/2011.

La CFFE (en inglés Physical Energy Flows Accounts -PEFA-) registra la interrelación con el medioambiente de los flujos de energía producidos y usados en las actividades económicas llevadas a cabo por las unidades residentes (y los hogares) de las economías nacionales. La cuenta tiene los mismos límites conceptuales que el Sistema Europeo de Cuentas Económicas (SEC), que se basa también en el principio de residencia ya enunciado.

Cabe señalar que la elaboración de la cuenta se basa en la ley de conservación de la energía, la cual establece que la energía no se crea, ni se destruye, solo se transforma. Por otra parte, al igual que en otras cuentas medioambientales físicas, en esta cuenta se incorpora el medioambiente como un sector de actividad económica más, en razón de que es el origen de los flujos de recursos energéticos y el destino de los residuos energéticos. Con el fin de permitir la comparación entre los valores de las estadísticas energéticas que están expresados en unidades de masa, volumen o energía, todas las magnitudes de la cuenta vienen referidas en terajulios (TJ).

La cuenta objeto de estudio registra los flujos físicos de energía que tienen lugar:

- Del medioambiente al sistema económico.

- En el ámbito del sistema económico.

- Del sistema económico (incluyendo los hogares) al medioambiente.

- Entre el sistema económico y el resto del mundo (importaciones y exportaciones).

Estos tipos de flujos físicos de energía están conformados respectivamente por las siguientes categorías de materiales energéticos:

- Recursos naturales energéticos: son aquéllos que son extraídos del medioambiente para ser usados en los procesos económicos de producción.

- Productos energéticos: son materiales que resultan del proceso de producción llevado a cabo por las unidades de actividad económica.

- Residuos energéticos: son materiales (sólidos, líquidos o gaseosos) descargados, vertidos o emitidos al medioambiente. En particular, los gases de combustión de productos energéticos se consideran residuos vertidos (emitidos) al medioambiente. 
En los flujos energéticos con el resto del mundo, los tres materiales energéticos citados pueden hacer acto de presencia tanto en las exportaciones como en las importaciones.

Los flujos físicos de energía, tanto en su origen (suministro/oferta) como en su destino (uso/demanda) pueden ser generados por cinco tipos de unidades contables:

- Ramas de actividad económica.

- Hogares como consumidores finales.

- Acumulación (variación de stocks de productos y de residuos en el sistema económico).

- Medioambiente.

- Resto del mundo.

Las Estadísticas Energéticas son la principal fuente de información para la elaboración de la CFFE. En el año 2004 se promulgó en el ámbito de la Unión Europea una Directiva obligando a sus Estados miembros a recopilar datos cuantitativos sobre la energía. Posteriormente, el Reglamento (CE) 1099/2008 estableció un marco común para la elaboración, transmisión, evaluación y difusión de estadísticas comparables sobre energía en armonía con lo establecido por la Agencia Internacional de la Energía. En España, las estadísticas energéticas son confeccionadas en la actualidad por el Ministerio para la Transición Ecológica y el Reto Demográfico, y son el resultado de la obtención y registro de datos administrativos sobre producción, importación, exportación y uso de productos energéticos. Sus principales fuentes son encuestas específicas al sector de la energía, estadísticas empresariales y del comercio internacional. Las estadísticas energéticas están conformadas por cinco bloques: carbón; petróleo; gas; electricidad; energías renovables.

Es importante señalar que para la elaboración de las Estadísticas Energéticas se sigue el principio de territorialidad, es decir que las estadísticas de la energía recopilan información sobre la energía producida y usada en un territorio nacional dado (definido por unas fronteras administrativas), tanto por las unidades residentes como por las no residentes. Por tanto, para pasar de estas estadísticas a la CFFE que se rige por el principio de residencia habrá que sumarles el uso de energía en el extranjero de las unidades residentes y restarles el uso de energía en el territorio nacional de las unidades no residentes.

Los materiales energéticos se pueden clasificar en productos primarios o secundarios. Se definen los primarios como aquéllos que se producen directamente mediante la extracción de recursos naturales energéticos del medioambiente, como pueden ser el petróleo crudo, el carbón mineral y el gas natural.

A su vez, los productos primarios pueden dividirse en combustibles de origen fósil y productos energéticos renovables. Los combustibles fósiles se extraen de los recursos naturales (carbón, petróleo crudo, gas natural, combustibles nucleares, etc.). Los productos energéticos renovables, a excepción de la energía geotérmica, se obtienen de los flujos de energía solar, eólica o gravitacional (mareas y olas). 
Por su parte, los productos secundarios resultan de la transformación de productos energéticos primarios u otros secundarios, en otros tipos de productos energéticos. La generación de electricidad quemando combustibles es un ejemplo de producto secundario. Otros ejemplos pueden ser los productos derivados del refino del petróleo bruto (gasolinas, fueloil, etc.), el coque obtenido a partir del carbón, el carbón vegetal a partir de la leña, etc.

Pueden existir usos no energéticos de productos energéticos (producción de plásticos, lubricantes, disolventes, asfaltos, parafinas, betún, etc.)

Los residuos energéticos pueden tener diversos orígenes:

- Residuos urbanos reciclables.

- Residuos urbanos e industriales no reciclables.

- Pérdidas de producto y energía durante la extracción, distribución, almacenamiento, transformación y disipación del calor, producidas por el uso final de la energía.

- Energía contenida en los productos de uso no energético (lubricantes, asfaltos, parafinas, betún, disolventes, etc.).

Las pérdidas de energía pueden producirse durante los procesos de transformación de productos primarios a partir de recursos naturales (refino del petróleo, quemas y fugas de gas natural, etc.) o por procesos de uso final (por ejemplo, calor disipado durante la quema de un combustible). También pueden producirse en la distribución de productos energéticos, como resultado de la evaporación y fuga de combustibles líquidos, pérdidas de calor durante el transporte de vapor o pérdidas durante la distribución de gas, transmisión de electricidad o transporte por tuberías.

\section{GRÁFICO 7}

\section{SÍNTESIS DE LA CLASIFICACIÓN DE LOS PRODUCTOS ENERGÉTICOS}

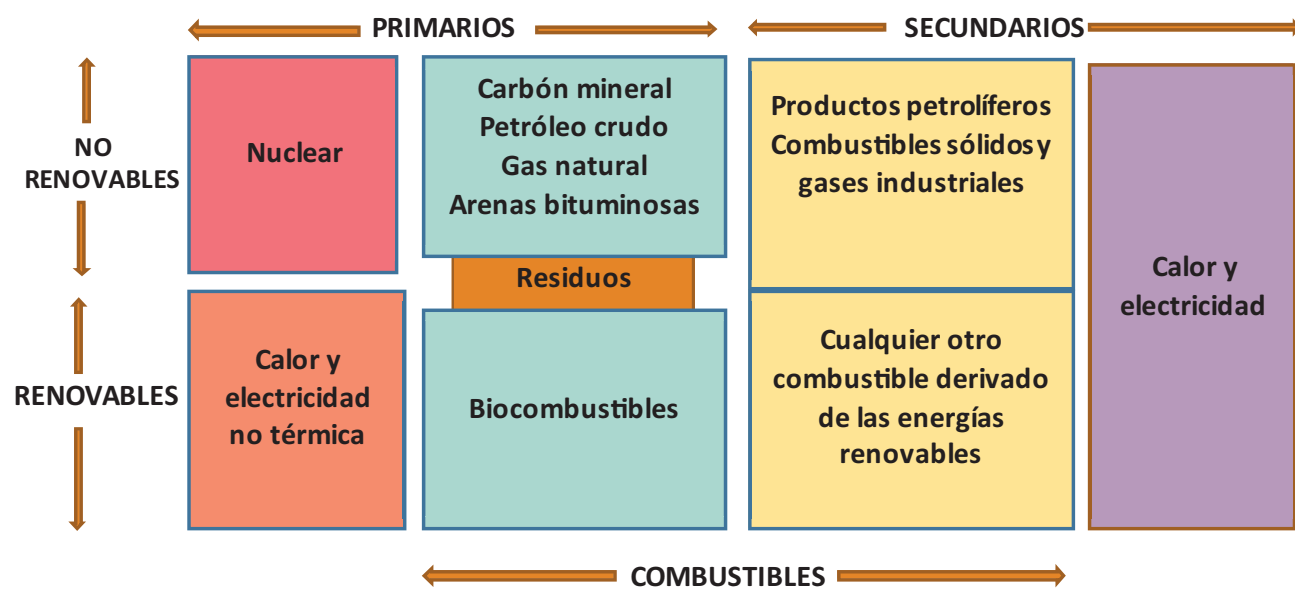

FUENTE: Elaboración propia a partir del Manual de Estadísticas Energéticas (Agencia Internacional de la Energía). 
En Cuadro 1 se especifican las identidades contables que deben cumplirse para convertir la información de las Estadísticas energéticas (principio de territorialidad) al formato de la Cuenta de los flujos físicos de energía (principio de residencia). Estos ajustes intervienen únicamente en las actividades de transporte, por ser las que pueden realizarse tanto en el país de residencia de la unidad económica como en el extranjero.

\section{CUADRO 1}

PASO DE LAS ESTADÍSTICAS ENERGÉTICAS A LA CUENTA DE FLUJOS FÍSICOS DE LA ENERGÍA

\begin{tabular}{|l|l|l|}
\hline $\mathbf{1}$ & & $\begin{array}{l}\text { Uso de energía por las unidades residentes y no residentes en el territorio } \\
\text { nacional (uso interior) (estadísticas energéticas) }\end{array}$ \\
\hline $\mathbf{2}$ & más & Uso de energía en el extranjero de las unidades residentes \\
\hline 2.1 & & PESCA EN AGUAS INTERNACIONALES* \\
\hline 2.2 & & TRANSPORTE TERRESTRE INTERNACIONAL** \\
\hline 2.3 & & TRANSPORTE MARÍTIMO INTERNACIONAL \\
\hline 2.4 & & TRANSPORTE AÉREO INTERNACIONAL \\
\hline $\mathbf{3}$ & menos & Uso de energía en el territorio nacional de las unidades no residentes* \\
\hline 3.1 & & TRANSPORTE TERRESTRE NACIONAL*** \\
\hline 3.2 & & TRANSPORTE MARÍTIMO NACIONAL \\
\hline 3.3 & & TRANSPORTE AÉREO NACIONAL \\
\hline $\mathbf{4}$ & igual a & $\begin{array}{l}\text { Uso de energía por las unidades residentes tanto en el territorio nacional } \\
\text { como en el extranjero (Cuenta de los flujos físicos de la energía) }\end{array}$ \\
\hline
\end{tabular}

NOTA: * Se considera que, en el sector de la pesca, el consumo de las unidades no residentes en territorio nacional no es significativo. ** Por «transporte internacional» se entiende el llevado a cabo en el extranjero, o entre un puerto o aeropuerto nacional y uno extranjero (y viceversa). *** Por «transporte nacional» se entiende el llevado a cabo en el ámbito territorial del país.

FUENTE: Elaboración propia a partir del Manual de la Cuenta de Flujos Físicos de la Energía (Eurostat).

\subsection{Metodología y estructura}

La cuenta se estructura en cuatro tablas principales que son un output directo de la macro «PEFA-builder»:

- Tabla A. Suministro de flujos de energía.

- Tabla B. Uso de flujos de energía.

- Tabla C. Flujos energéticos más relevantes para las emisiones.

- Tabla D. Vectores de indicadores energéticos clave.

Las dos primeras constituyen el núcleo central de la cuenta mientras que los dos restantes son tablas auxiliares que sirven tanto para visualizar los ajustes de residencia, como para calcular las magnitudes e indicadores derivados de la cuenta. 
Las Tablas A y B tienen el mismo formato matricial, y siguen el diseño de las tablas origen/destino del marco input/output registradas en el SEC. En filas figuran los flujos de materiales energéticos, y en columnas los diferentes tipos de operación de dichos flujos: producción y consumos intermedios (unidades económicas), consumo final (hogares), variación de activos/stocks (acumulación), resto del mundo (importaciones/exportaciones), flujos de suministro de recursos naturales y destino de los residuos (medioambiente).

\section{TABLAS A Y B DE LA CUENTA DE FLUJOS FÍSICOS DE ENERGÍA}

TABLA A. SUMINISTRO DE FLUJOS DE ENERGÍA

\begin{tabular}{|c|c|c|c|c|c|c|}
\hline & $\begin{array}{l}\text { Ramas de } \\
\text { actividad } \\
\text { económica }\end{array}$ & Hogares & $\begin{array}{l}\text { Acumula- } \\
\text { ción }\end{array}$ & $\begin{array}{c}\text { Resto del } \\
\text { mundo } \\
\text { (Importa- } \\
\text { ciones) }\end{array}$ & $\begin{array}{l}\text { Medio- } \\
\text { ambiente }\end{array}$ & Total \\
\hline $\begin{array}{l}\text { Recursos } \\
\text { naturales }\end{array}$ & & & & & A & \\
\hline Productos & $\mathrm{C}$ & & & $\mathrm{D}$ & & \\
\hline Residuos & I & $\mathrm{J}$ & K & $\mathrm{L}$ & M & \\
\hline
\end{tabular}

TABLA B. USO DE FLUJOS DE ENERGÍA

\begin{tabular}{|c|c|c|c|c|c|c|}
\hline & $\begin{array}{c}\text { Ramas de } \\
\text { actividad } \\
\text { económica }\end{array}$ & Hogares & $\begin{array}{c}\text { Acumula- } \\
\text { ción }\end{array}$ & $\begin{array}{c}\text { Resto del } \\
\text { mundo } \\
\text { (Exporta- } \\
\text { ciones) }\end{array}$ & $\begin{array}{c}\text { Medio- } \\
\text { ambiente }\end{array}$ & Total \\
\hline $\begin{array}{c}\text { Recursos } \\
\text { naturales }\end{array}$ & $\mathrm{B}$ & & & & & \\
\hline Productos & $\mathrm{E}$ & $\mathrm{F}$ & $\mathrm{G}$ & $\mathrm{H}$ & & \\
\hline Residuos & $\mathrm{N}$ & & $\mathrm{O}$ & $\mathrm{P}$ & $\mathrm{Q}$ & \\
\hline
\end{tabular}

FUENTE: Elaboración propia a partir del Manual de la Cuenta de Flujos Físicos de la Energía (Eurostat).

En esta cuenta, el desglose estadístico de las unidades económicas se establece en 21 ramas de actividad económica, según las divisiones a dos dígitos de la Clasificación Nacional de Actividades Económicas CNAE-2009. La intersección de las columnas con las filas conforma las submatrices que asocian los flujos de productos a un suministrador o usuario dado.

La metodología de la cuenta establece una serie de convenios de tal manera que algunas de las submatrices (fondo de color grisáceo) de las Tablas A y B, no pueden contener valor alguno, es decir, que la combinación de las dos dimensiones (filas y columnas) no son conceptualmente factibles. Los convenios más relevantes establecidos por el Manual Physical Energy Flow Accounts (Eurostat, 2014) son: 
- Los recursos naturales energéticos solo pueden ser suministrados por el medioambiente y usados por las ramas de actividad económica.

- Las actividades económicas que los hogares lleven a cabo se asignarán a la rama de actividad económica que corresponda y no a los hogares.

- El medioambiente no puede suministrar ni usar productos energéticos, y no puede usar otros residuos energéticos que no sean las pérdidas de energía.

La Tabla A establece el suministro de recursos naturales, productos y residuos energéticos (filas) según su origen (columnas). Por otra parte, y desde la perspectiva de las unidades contables (columnas), estos flujos pueden considerarse como las «salidas» de flujos de energía desde dichas unidades. La Tabla B por su parte establece el uso de los recursos naturales, productos y residuos energéticos (filas) según su destino (columnas), es decir su usuario. Por otra parte, y desde la perspectiva de las unidades contables (columnas), estos flujos pueden considerarse como las «entradas» de flujos de energía en dichas unidades. Cabe señalar que, en el caso del medioambiente, el vocablo «uso» puede no ser el más adecuado ya que la naturaleza no «usa» residuos energéticos, sino que los recibe haciendo las veces de sumidero.

Las submatrices de las Tablas A y B se pueden estructurar en cuatro bloques según los tipos de flujos energéticos y de suministrador/usuario.

- Cómo se extraen y se usan los recursos naturales energéticos y como se suministran los productos energéticos):

A. Recursos naturales suministrados por el medioambiente.

B. Recursos naturales extraídos por las ramas de actividad.

C. Suministro de productos por las unidades económicas.

D. Suministro de materiales provenientes del resto del mundo (importaciones).

- Cómo se usan los productos energéticos:

E. Consumos intermedios de productos de las ramas de actividad.

F. Uso de productos por los hogares.

G. Incrementos y cambios netos de stocks de activos producidos.

H. Uso de materiales por el resto del mundo (exportaciones).

- Cómo se generan los residuos energéticos:

I. Residuos generados por las ramas de actividad.

J. Residuos generados por los hogares.

K. Disminuciones de activos producidos o de energía de los stocks (activos o existencias) (demoliciones, desguaces, achatarramiento).

L. Residuos provenientes del resto del mundo (importaciones).

M. Suministro de residuos desde el medioambiente (ej. recogida de vertidos de hidrocarburos).

- Cómo se usan los residuos energéticos:

N. Recogida y tratamiento de residuos por las ramas de actividad. 
O. Incrementos /acumulación de residuos (ej. vertederos o energía incorporada a los residuos).

P. Residuos enviados al resto del mundo (exportaciones).

Q. Recepción de residuos por el medioambiente.

\subsection{Elaboración}

En la medida de sus posibilidades técnicas y del estadio de su información estadística, cada país puede implantar las herramientas informáticas para, a partir de las estadísticas energéticas, elaborar la CFFE. No obstante, Eurostat ha puesto a disposición de sus países miembros una macro-«PEFA-builder»- que permite elaborar la CFFE de una manera secuencial y que es utilizada a dichos efectos por el INE.

Para aplicar los ajustes de residencia, es decir para pasar del principio de territorialidad (estadísticas energéticas) al de residencia (cuenta), se utiliza información estadística auxiliar, como datos de origen administrativo sobre trayectos de las compañías aéreas y número de pasajeros por kilómetro, tonelaje de los buques, encuestas sobre la actividad pesquera, parque de vehículos, etc. También se puede utilizar la información que sobre consumos, importaciones y exportaciones de combustibles figura en las tablas de origen-destino (input-output) que se elaboran en el marco del SEC, estadísticas de comercio exterior, etc.

Ahora bien, el output del «PEFA-builder» presenta la información según agregaciones de ramas de Clasificación Nacional de Actividades Económicas (CNAE2009), por lo que es necesario efectuar algunas desagregaciones de los mismos. Para ello, se suelen utilizar operaciones estadísticas que lleva a cabo la Oficina Central de Estadística del país (encuestas de empresas y de productos industriales, encuestas de consumos energéticos, estadísticas sobre generación, recogida y tratamiento de residuos, encuestas de presupuestos familiares, tablas origen-destino de la Contabilidad Nacional, etc.).

Por otra parte, en el caso del transporte por carretera, muchos establecimientos (industriales o del sector servicios) consumen combustibles en actividades de transporte que tienen el carácter de servicios auxiliares. En ese caso, dichos consumos de energía (así como las cantidades de residuos generadas) deben ser asignados a la división de la CNAE que corresponde a la actividad principal del establecimiento.

\subsection{Estimaciones e indicadores}

Seguidamente, en los Cuadros 2, 3 y 4 se presentan las estimaciones de la CFFE con referencia temporal de los datos 2018. Como ya se ha indicado, la unidad de medida que se utiliza en la cuenta es el terajulio (TJ), debiéndose de aplicar coeficientes de conversión estandarizados a las unidades que se utilizan en cada uno de los cinco bloques de productos energéticos que conforman las Estadísticas Energéticas. Así 
en lo que respecta al carbón, un TJ equivale a 34,121 toneladas equivalentes en carbón, en el caso del petróleo, un TJ equivale a 23,884 toneladas equivalentes de petróleo; en el caso la electricidad y de las energías renovables, un TJ equivale a 0,2777 gigavatios hora; en el caso del gas, la equivalencia depende del calor calorífico del gas considerado.

La energía que suministran las ramas económicas y las importaciones representa el $66,1 \%$ del total (productos energéticos primarios o secundarios); los hogares y las ramas económicas generan el $27,0 \%$ del total en forma de residuos energéticos y el $6,9 \%$ son recursos naturales provenientes del medioambiente.

\section{CUADRO 2}

ORIGEN DE LA ENERGÍA (2018)

(En miles de terajulios)

\begin{tabular}{|l|c|c|c|}
\hline & Total & $\begin{array}{c}\text { \% sobre } \\
\text { el total }\end{array}$ & $\begin{array}{c}\text { \% variación } \\
\text { anual }\end{array}$ \\
\hline Recursos naturales energéticos & $1.384,70$ & 6,9 & $-0,3$ \\
\hline Productos energéticos & $13.263,00$ & 66,1 & $-0,8$ \\
\hline Residuos energéticos & $5.421,40$ & 27,0 & 2,2 \\
\hline TOTAL & $\mathbf{2 0 . 0 6 9 , 1 0}$ & $\mathbf{1 0 0 , 0}$ & $\mathbf{0 , 0}$ \\
\hline
\end{tabular}

FUENTE: INE.

En lo que respecta al destino de la energía, se observa que más de la mitad de los flujos $(58,0 \%)$ son usados por las ramas de actividad económica, el 6,6\% por los hogares y el 9,0\% por las exportaciones. El medioambiente usa (recibe) el $25,7 \%$ en su mayor parte en forma de calor disipado por la combustión de productos energéticos.

\section{CUADRO 3}

DESTINO DE LA ENERGÍA (2018)

(En miles de terajulios)

\begin{tabular}{|l|c|c|c|}
\hline & Total & $\begin{array}{c}\text { \% sobre } \\
\text { el total }\end{array}$ & $\begin{array}{c}\text { \% variación } \\
\text { anual }\end{array}$ \\
\hline Ramas de actividad & $11.637,2$ & 58,0 & 0,6 \\
\hline Hogares & $1.323,6$ & 6,6 & 0,4 \\
\hline Exportaciones & $1.809,6$ & 9,0 & $-2,0$ \\
\hline Medioambiente (pérdidas de energía) & $5.158,5$ & 25,7 & 0,7 \\
\hline V.E y ajustes* & 140,2 & 0,7 & - \\
\hline TOTAL & $\mathbf{2 0 . 0 6 9 , 1}$ & $\mathbf{1 0 0 , 0}$ & $\mathbf{0 , 0}$ \\
\hline
\end{tabular}

NOTA: * V.E (variación de existencias) y ajustes estadísticos.

FUENTE: INE. 
La Cuenta de Flujos Físicos de la Energía posibilita también establecer un balance comercial físico (importaciones menos exportaciones) de productos energéticos. En el Cuadro 4 se observa que el balance para España es negativo, aunque en los epígrafes de productos del petróleo y de combustibles es positivo.

\section{CUADRO 4}

COMPONENTES DEL BALANCE COMERCIAL FÍSICO DE PRODUCTOS ENERGÉTICOS (2018)

(En miles de terajulios)

\begin{tabular}{|l|r|r|r|r|r|}
\hline & $\begin{array}{c}\text { Balance } \\
\text { comercial } \\
\text { físico }\end{array}$ & $\begin{array}{c}\text { Importa- } \\
\text { ciones }\end{array}$ & $\begin{array}{c}\text { \% sobre } \\
\text { el total }\end{array}$ & $\begin{array}{c}\text { Exporta- } \\
\text { ciones }\end{array}$ & $\begin{array}{c}\text { \% sobre } \\
\text { el total }\end{array}$ \\
\hline Productos de la industria extractiva & $-4.401,0$ & $4.526,0$ & 80,0 & 124,9 & 6,9 \\
\hline Coque y productos del refino del petróleo & 569,8 & 978,9 & 17,3 & $1.548,7$ & 85,6 \\
\hline Biocombustibles & 19,4 & 70,1 & 1,2 & 89,5 & 4,9 \\
\hline Energía eléctrica y calor & $-40,0$ & 86,4 & 1,5 & 46,5 & 2,6 \\
\hline TOTAL & $\mathbf{- 3 . 8 5 1 , 8}$ & $\mathbf{5 . 6 6 1 , 4}$ & $\mathbf{1 0 0 . 0}$ & $\mathbf{1 . 8 0 9 , 6}$ & $\mathbf{1 0 0 , 0}$ \\
\hline
\end{tabular}

FUENTE: INE.

En lo que respecta la elaboración de indicadores energéticos, en base a la información de las tablas A y B se pueden calcular siete indicadores clave que, si bien tienen un carácter complementario, resultan muy útiles para seguimiento de ciertos aspectos como puede ser el de la medición de las emisiones de gases procedentes del uso de la energía, asignando a cada producto energético un volumen de emisiones por unidad de energía usada. Estos factores de emisión (o coeficientes técnicos de emisión) por unidad de producto, dependen también de la rama de actividad económica que consuma el recurso o producto energético. Los siete indicadores clave de uso de la energía que la CFFE permite elaborar son:

- Extracción de recursos energéticos naturales por las ramas de actividad económica (columna de totales en la submatriz B).

- Producción de productos energéticos por las ramas de actividad económica (columna de totales en la submatriz C).

- Consumo intermedio total de productos energéticos por ramas de actividad económica (columna de totales de la submatriz E).

- Uso total de productos energéticos por los hogares (total de la submatriz F).

- Uso total de los residuos con fines energéticos (columna de totales de la submatriz N).

- Uso neto de energía (suma de las columnas de las submatrices I, J, y O).

- Input / output energético total (columna de totales de las submatrices C, I y J de la Tabla A que es igual a la columna de totales de las submatrices B, E, F y $\mathrm{N}$ de la Tabla B). 


\section{Cuenta de emisiones a la atmósfera}

\subsection{Marco conceptual}

Las Cuentas de Emisiones a la Atmósfera (CEA) -Air Emission Accounts(AEA, en sus siglas en inglés) son unas cuentas ambientales que forman parte del Sistema de Contabilidad Económica y Medioambiental (System of Environmental Economic Accounting, SEEA, en sus siglas en inglés). Son uno de los seis módulos del Reglamento (UE) 691/2011del Parlamento Europeo y del Consejo ${ }^{4}$, de 6 de julio de 2011, relativo a las cuentas económicas europeas medioambientales, y su objetivo principal es proporcionar importante información a la Unión Europea para poder tomar decisiones en materia de políticas de cambio climático, crecimiento verde y productividad de los recursos.

Las CEA presentan los datos sobre las emisiones contaminantes a la atmósfera desglosados por la actividad económica emisora y el sector hogares como consumidores finales, de acuerdo con la metodología para las Cuentas Nacionales del Sistema Europeo de Cuentas Nacionales y Regionales (ESA), siendo compatible con el Sistema Internacional de Cuentas Nacionales de las Naciones Unidas (SNA).

En las CEA cuando se habla de «emisiones a la atmósfera» se hace referencia a los flujos físicos de materiales gaseosos o a las partículas que se originan en el sistema económico (procesos productivos y/o de consumo), que son liberados a la atmósfera y que permanecen suspendidos en el aire durante un periodo sustancial. Como todos los residuos, las emisiones a la atmósfera son producciones secundarias y no deseadas, generadas en los procesos productivos y de consumo por los elementos del sistema económico. La mayoría de estos residuos se encuentran en un estado gaseoso, pero las partículas pequeñas $\left(\mathrm{PM}_{2.5}\right.$ y $\left.\mathrm{PM}_{10}\right)$ y los metales pesados son sólidos que permanecen en suspensión en la atmósfera durante periodos sustanciales y tienen ciertos comportamientos similares a los gases.

La lista de gases y de partículas que podrían ser registradas es enorme, pero en las CEA solo se solicita una limitada parte de estos. Se incluyen tanto las emisiones de los gases de efecto invernadero $\left(\mathrm{CO}_{2}\right.$, dióxido de carbono; $\mathrm{CH}_{4}$, metano; $\mathrm{N}_{2} \mathrm{O}$, óxido nitroso; HFC, hidrofluorocarbonos; $\mathrm{PFC}$, perfluorocarbonos; y $\mathrm{SF}_{6}$, hexafluoruro de azufre), como las emisiones de contaminantes del aire y responsables de la lluvia ácida (SOx, óxidos de azufre; $\mathrm{NO}_{\mathrm{x}}$, óxidos de nitrógeno; $\mathrm{NH}_{3}$, amoniaco), así como las de los precursores de la capa de ozono ( $\mathrm{CO}$, monóxido de carbono, y los ya mencionados $\mathrm{HFC}$, PFC y $\mathrm{SF}_{6}$ ), las de los gases responsables de la contaminación fotoquímica (COVNM, compuestos orgánicos volátiles -excepto metano-; y $\mathrm{NO}_{\mathrm{x}}$ ), y las emisiones de las partículas $\left(\mathrm{PM}_{10}\right.$, partículas de diámetro aerodinámico inferior a 10 micras; y $\mathrm{PM}_{2.5}$, partículas de diámetro aerodinámico inferior a 2,5 micras).

\footnotetext{
${ }^{4}$ https://eur-lex.europa.eu/legal-content/ES/TXT/PDF/?uri=CELEX:32011R0691\&from=EN
} 


\subsection{Metodología y estructura}

El total de residuos procedentes de las actividades económicas se denomina flujo bruto. Parte de este flujo es capturado, procesado y transformado por otras actividades económicas, reutilizándose como un recurso. Así pues, el flujo neto es la cantidad de emisiones atmosféricas que realmente atraviesan el límite del sistema, es decir, que terminan en el sistema ambiental.

Las CEA registran los flujos netos de materiales residuales gaseosos y de partículas en el aire que proceden de la economía nacional, es decir, de las actividades de producción y de consumo de unidades residentes, cruzan la frontera del sistema funcional entre economía y medioambiente, y pueden acabar en la atmósfera del territorio nacional o en la del resto del mundo. Esto implica que no todas las emisiones atmosféricas se tienen en consideración para incluirlas en las CEA.

En las Cuentas de emisiones a la atmósfera la frontera del sistema se define funcionalmente (sistema económico versus sistema ambiental) y no geográficamente (sistema nacional versus sistema del resto del mundo).

Después de haber cruzado esta frontera del sistema, las sustancias emitidas están fuera de cualquier control humano y se convierten en parte de los ciclos de materiales naturales y pueden generar diversos tipos de impactos ambientales. Si bien, en la práctica, existen algunos casos en los que el trazado de esta frontera del sistema funcional no es sencillo de definir y donde se deben adoptar ciertos acuerdos teóricos y técnicos. Eso ocurre, por ejemplo, con las emisiones a la atmósfera procedentes de plantas y bosques cultivados (y con su captación de las emisiones) o con las emisiones atmosféricas de vertederos, entre otros. Estos casos tienen en común que se refieren a las emisiones a la atmósfera procedentes de determinados orígenes (suelos agrícolas, humanos, plantas cultivadas y bosques, animales y vertederos) para las que se debe responder a la pregunta: ¿ se consideran parte del sistema económico o parte del sistema ambiental? Si son parte del sistema económico, deben registrarse de acuerdo con las reglas generales de las Cuentas Nacionales, ahora bien, si son parte del sistema ambiental, no deben registrarse en las CEA. La solución a esta cuestión consiste en la aplicación de dos reglas: en primer lugar aplicar los principios de las Cuentas Nacionales, lo que implica que cualquier emisión neta de gases y de partículas procedente de alguna actividad económica o humana debe ser registrada e incluida en las CEA (conforme a esta regla se debería incluir la respiración humana y la evapotranspiración); y en segundo lugar, y debido a consideraciones prácticas, sólo se consideran las emisiones netas a la atmósfera medibles que realmente se registran en los inventarios de emisiones y que se toman en consideración para calcular los totales de emisiones nacionales. 


\section{CUADRO 5}

ESTRUCTURA DE LAS CUENTAS DE EMISIONES A LA ATMÓSFERA

\begin{tabular}{|c|c|c|c|c|c|}
\hline Emisiones a la atmósfera & 1995 & 1996 & ... & 2014 & 2015 \\
\hline Ramas de actividad económica & \multirow{2}{*}{\multicolumn{5}{|c|}{$\begin{array}{l}\text { Emisiones a la atmósfera por actividad } \\
\text { económica }\end{array}$}} \\
\hline Clasificación CNAE (64 ramas) & & & & & \\
\hline Hogares (como consumidores finales), Total & \multirow{4}{*}{\multicolumn{5}{|c|}{$\begin{array}{l}\text { Emisiones a la atmósfera de los } \\
\text { hogares }\end{array}$}} \\
\hline Transporte & & & & & \\
\hline Calefacción & & & & & \\
\hline Otros & & & & & \\
\hline Totales (CEA) & \multicolumn{5}{|c|}{ TOTAL CEA } \\
\hline (-) Uds. residentes en el extranjero & \multirow{3}{*}{\multicolumn{5}{|c|}{ Tabla puente }} \\
\hline (+) Unidades NO residentes en el territorio nacional & & & & & \\
\hline$(+/-)$ Otros & & & & & \\
\hline $\begin{array}{l}\text { Total nacional conforme a UNFCCC o CLRTAP excluyendo } \\
\text { LULUCF }\end{array}$ & \multicolumn{5}{|c|}{ TOTAL INVENTARIO EMISIONES } \\
\hline
\end{tabular}

FUENTE: Elaboración propia.

En las columnas se incluyen los años de referencia para los cuales se reportan las emisiones a la atmósfera, comenzando en el año 1995 y hasta $n-2$ (siendo $n$ el año en que se realiza la recopilación de datos). Así, en 2021 se deben publicar los datos disponibles hasta 2019. El cuestionario de las CEA incluye toda la serie temporal, ya que una de las principales fuentes de información, los inventarios nacionales de emisiones, pueden ser revisados y modificados todos los años, y estos cambios pueden afectar a todo el periodo registrado.

En las filas se incluyen las actividades económicas responsables de las emisiones contaminantes, en concreto las 64 ramas de actividad económica conforme a la clasificación CNAE-20095 y los hogares como consumidores finales. Las emisiones se desglosan en tres subclases, las emisiones del transporte, las emisiones procedentes de la calefacción y una tercera categoría que incluye el resto de emisiones bajo el epígrafe de otros, para terminar con los totales de las CEA, los elementos de la tabla puente y los totales nacionales procedentes de los inventarios de emisiones y de otras publicaciones oficiales de emisiones, principal fuente de información para la elaboración de las CEA.

\footnotetext{
5 https://www.ine.es/dyngs/INEbase/es/operacion.htm?c=Estadistica_C\&cid=1254736177032\&menu= ultiDatos \&idp=1254735976614
} 


\subsection{Elaboración}

La elaboración de las CEA, como ya hemos indicado, se desarrolla a partir de una extensa variedad de fuentes de información existentes. La fuente de información más importante de todas es el Inventario Nacional de Emisiones, pero también se utilizan otras fuentes de información, como por ejemplo los datos sobre el uso de la energía procedentes de las estadísticas y de los balances energéticos, los Cuentas Nacionales publicadas por el INE, estadísticas del agua, estadísticas y registros de aviación, estadísticas de flota pesquera, estadísticas de tráfico rodado, registro de vehículos y matriculaciones, estadísticas de transporte de mercancías y pasajeros, etc. Los datos procedentes de las distintas fuentes de información deben ser adaptados y reorganizados de acuerdo con los principios y clasificaciones contables de las Cuentas Nacionales; este proceso incluye la reorganización de los datos de acuerdo con las actividades económicas de las Cuentas Nacionales (industrias y hogares), la eliminación de cualquier doble contabilización y la suma o la resta (según corresponda) de datos en las tablas puente para que los límites del sistema se correspondan con los límites del sistema de las Cuentas Nacionales.

Como ya se comentó en la introducción a las cuentas ambientales físicas, una de las principales diferencias entre las estadísticas y los balances de energía y los inventarios de emisiones, por una parte, y las Cuentas Nacionales, por otra, está relacionada con la diferencia entre la definición geográfica y la definición económica de un país. Las estadísticas y los balances de energía, así como los inventarios nacionales de emisiones utilizan una definición geográfica o de territorio nacional, mientras que las Cuentas Nacionales se basan en el principio de residencia. Básicamente, para convertir un sistema de emisiones geográfico en un sistema económico, las cantidades de contaminantes emitidas por las unidades no residentes que operan en el territorio nacional necesitan ser eliminadas, y es necesario añadir las emisiones de las unidades residentes que operan en el resto del mundo (Gráfico 8).

Para elaborar las CEA se pueden distinguir dos enfoques distintos, el enfoque del inventario de emisiones y el enfoque de la energía. El «enfoque del inventario de emisiones» es aquel en el que se parte de la información disponible en los inventarios nacionales de emisiones existentes y conlleva reorganizar esos datos para adoptarlos a un formato compatible con el de las Cuentas Nacionales. Es importante señalar que los totales de las CEA de la economía nacional probablemente diferirán de los totales de los inventarios nacionales de emisiones debido a los distintos principios de medición utilizados en cada uno de ellos (principio de emisión geográfico en los inventarios y principio de residencia en las CEA). De existir estas diferencias, las mismas se registran y se presentan en las denominadas «tablas puente». $\mathrm{El}$ «enfoque de la energía», es aquel en el que se parte de las estadísticas y saldos de energía que se remiten a las Cuentas de la energía, a partir de las cuales se calculan las emisiones atmosféricas utilizando determinados factores de emisión. Las Cuentas de emisiones a la atmósfera elaboradas por el INE, siguen el enfoque del inventario de emisiones. 


\section{GRÁFICO 8}

\section{INTERRELACIÓN ENTRE EL PRINCIPIO DE RESIDENCIA Y EL DE TERRITORIALIDAD}

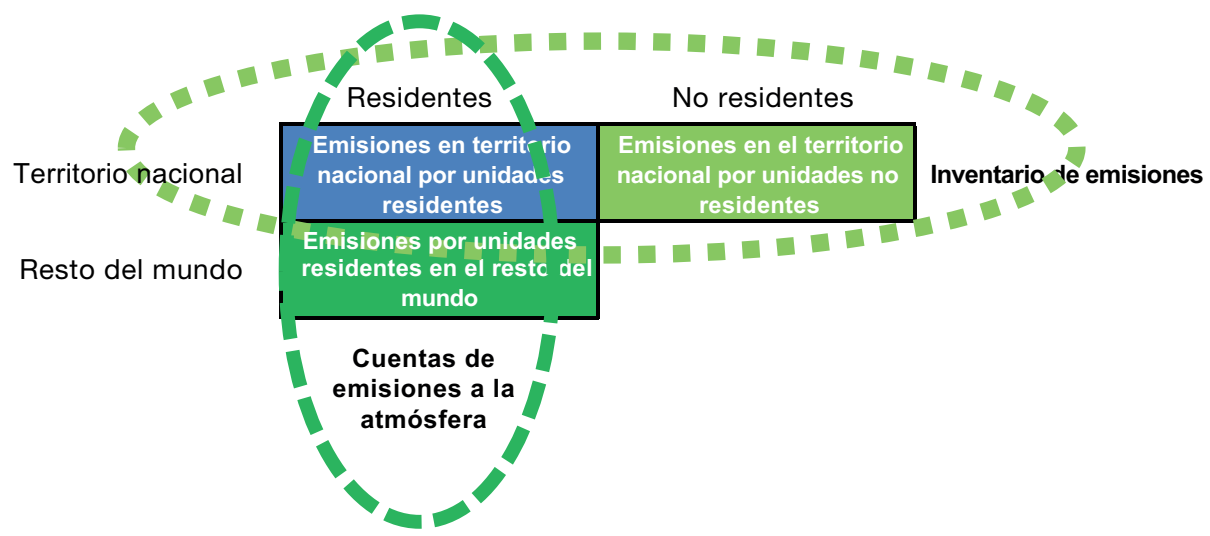

FUENTE: Elaboración propia.

A la hora de elaborar las CEA utilizando los inventarios hay que tener en cuenta alguna de sus características más relevantes como, por ejemplo, que los datos de emisiones se refieren al territorio geográfico del país de referencia y que los datos de emisiones se desglosan conforme a clasificaciones técnicas orientadas al proceso que genera la emisión. Por el contrario, una característica básica de las CEA es que las emisiones deben relacionarse con las actividades de las unidades económicas residentes que forman parte de la economía nacional (principio de residencia) y el desglose de los datos debe ser coherente con las clasificaciones adoptadas para las actividades económicas (CNAE). Por tanto, cuando se elaboran las CEA siguiendo el «enfoque del inventario» se necesita llevar a cabo dos ajustes, la reclasificación y adaptación de los datos del inventario a una clasificación de actividad económica (CNAE-2009) y la estimación de las emisiones generadas por las unidades económicas residentes a partir de las emisiones generadas por todas las fuentes de emisión (económicas y no económicas) en el territorio geográfico nacional.

Los datos de las CEA se desglosan por actividades económicas, en concreto, y como ya se ha indicado, en 64 ramas de actividad conforme a la clasificación CNAE-2009 y además se incluyen las emisiones de las actividades de consumo final de los hogares. Al utilizar como fuente de información primaria los inventarios nacionales de emisiones y las estadísticas energéticas, aparece el problema de que esta información se encuentra organizada en base a otros tipos de clasificación diferentes, por lo que es imprescindible asignar y distribuir los datos originales entre las actividades económicas de las CEA. Así pues, para asignar las emisiones a las actividades económicas se deben seguir tres pasos. En primer lugar, se debe entender la clasificación de los datos de las fuentes primarias (inventario de emisiones y estadísticas de la energía). En segundo lugar, se debe comprender cómo 
se delimitan y cómo se registran las actividades de producción y consumo de las unidades residentes en las tablas las CEA conforme a la clasificación CNAE-2009. Y finalmente, es imprescindible desarrollar unas tablas de correspondencia específica con los ajustes necesarios para asignar y estimar los valores de las emisiones de las CEA, para lo que se puede utilizar de información auxiliar. El objetivo es desarrollar las tablas que establezcan las correspondencias para poder asignar y distribuir los datos de las fuentes de información primarias, organizados conforme a sus propias clasificaciones, a las categorías de actividad de la CNAE y a los hogares como consumidores finales. En general, se pueden distinguir dos tipos de correspondencias, bien una correspondencia de uno a uno entre una categoría de la fuente de datos original y la categoría CNAE o bien una correspondencia de uno a varios entre una categoría de origen de datos original y varias categorías NACE.

Para muchas de las categorías de los datos de las fuentes de información original puede haber una correspondencia directa entre la categoría original y las categorías de la CNAE. Sin embargo, para varias de las categorías de datos originales existe una correspondencia de una a varias actividades económicas, siendo necesario hacer uso de información auxiliar para poder estimar la distribución de los datos. Los datos auxiliares pueden comprender datos físicos (por ejemplo, uso de energía o propiedad del vehículo), datos de empleo y/o datos monetarios que proporcionen información adicional que ayude a estimar la correspondencia. También se pueden utilizar agregados de Cuentas Nacionales, tales como la producción y el valor añadido.

\subsection{Estimaciones e indicadores}

En 2019 la economía española emitió 323,2 millones de toneladas de gases de efecto invernadero, un 5,7\% menos que en 2018. En el Gráfico 9 se incluye el total de emisiones desde el año 2008, donde se observa un marcado descenso de las mismas hasta 2013 y una estabilización con ligeras variaciones al alza o a la baja desde entonces y hasta 2019.

Del total de emisiones de gases de efecto invernadero en 2019, la industria manufacturera concentró el 25,0\% del total, emitiendo 80,8 millones de toneladas de $\mathrm{CO}_{2}$ e equivalentes. Los hogares emitieron 68,4 millones de toneladas de $\mathrm{CO}_{2} \mathrm{e}$, un $21,2 \%$ del total de la economía, y el suministro de energía eléctrica, gas, vapor, aire acondicionado y agua emitió 58,2 millones de toneladas de $\mathrm{CO}_{2}$, un $18 \%$ (Cuadro 6).

Las mayores cantidades de dióxido de carbono emitidas correspondieron a la industria manufacturera (75,0 millones de toneladas), a los hogares (65,6 millones) y al suministro de energía eléctrica, gas, vapor, aire acondicionado y agua $(45,4$ millones). En conjunto, estos tres sectores representaron el 71,5\% del total de las emisiones de dióxido de carbono a la atmósfera (Gráfico 10). 


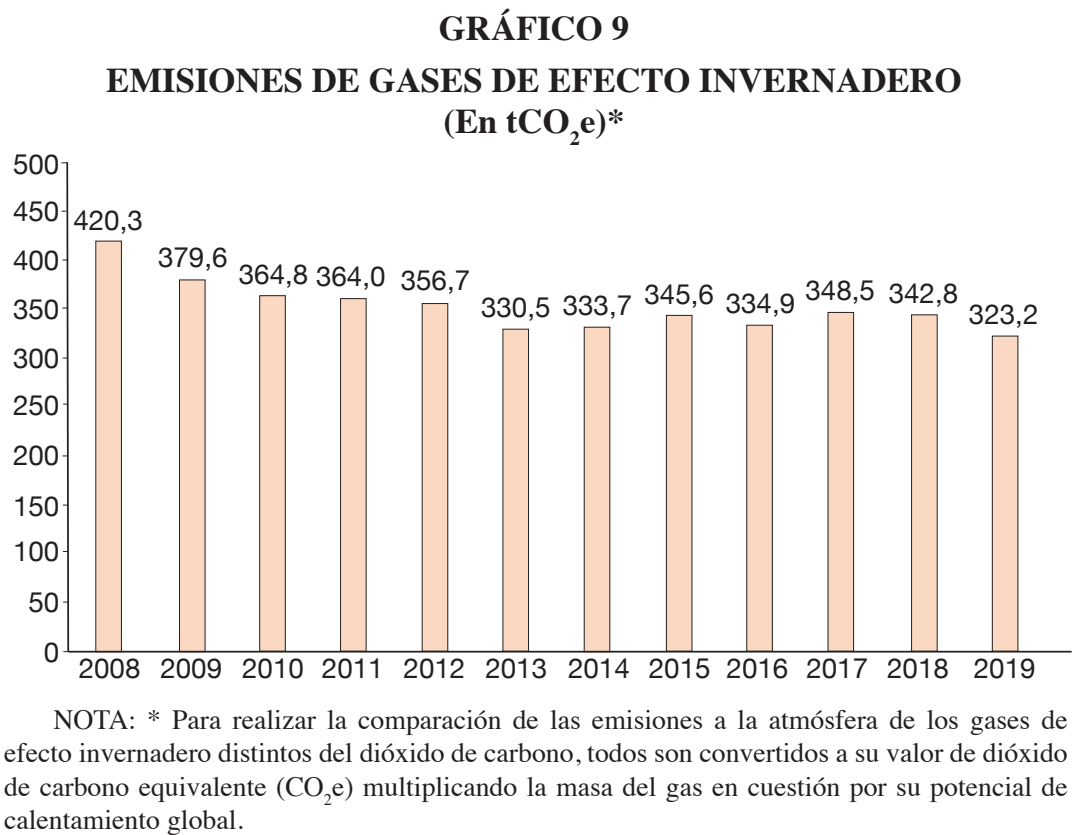

FUENTE: INE.

\section{CUADRO 6}

EMISIONES DE GASES DE EFECTO INVERNADERO POR RAMAS DE ACTIVIDAD Y HOGARES (2019)

$\left(\mathrm{En} \mathrm{tCO} \mathrm{C}_{2} \mathrm{e}\right)$

\begin{tabular}{|l|c|c|r|r|r|r|}
\hline & $\begin{array}{c}\text { Total } \\
\mathbf{G E I}\end{array}$ & $\begin{array}{c}\text { Variación } \\
\text { anual } \\
\mathbf{( \% )}\end{array}$ & $\begin{array}{c}\text { Dióxido de } \\
\text { carbono } \\
\left(\mathbf{C O}_{\mathbf{2}}\right)\end{array}$ & $\begin{array}{c}\text { Metano } \\
\left(\mathbf{C H}_{4}\right)\end{array}$ & $\begin{array}{c}\text { Óxido } \\
\text { nitroso } \\
\left(\mathbf{N}_{\mathbf{2}} \mathbf{O}\right)\end{array}$ & $\begin{array}{c}\text { Otros } \\
\text { GEI }\end{array}$ \\
\hline Industria manufacturera & $80.785,3$ & $-5,8$ & $74.961,8$ & $2.407,2$ & 619,3 & $2.797,0$ \\
\hline $\begin{array}{l}\text { Suministro de energía } \\
\text { eléctrica, gas, vapor, aire } \\
\text { acondicionado y agua }\end{array}$ & $58.215,9$ & $-19,4$ & $45.384,4$ & $10.862,8$ & $1.566,8$ & 401,9 \\
\hline $\begin{array}{l}\text { Agricultura, ganadería, } \\
\text { selvicultura y pesca }\end{array}$ & $50.971,0$ & $-1,6$ & $12.080,6$ & $25.306,7$ & $13.562,5$ & 21,2 \\
\hline $\begin{array}{l}\text { Transporte y } \\
\text { almacenamiento }\end{array}$ & $45.261,0$ & 2,1 & $44.580,4$ & 39,9 & 525,6 & 115,1 \\
\hline Resto de ramas de actividad & $19.554,0$ & $-2,5$ & $17.378,3$ & 294,9 & 483,3 & $1.397,5$ \\
\hline Hogares & $68.433,7$ & $-0,2$ & $65.602,8$ & 899,9 & 627,5 & $1.303,5$ \\
\hline TOTAL & $\mathbf{3 2 3 . 2 2 0 , 9}$ & $-\mathbf{5 , 7}$ & $\mathbf{2 5 9 . 9 8 8 , 3}$ & $\mathbf{3 9 . 8 1 1 , 4}$ & $\mathbf{1 7 . 3 8 5 , 0}$ & $\mathbf{6 . 0 3 6 , 2}$ \\
\hline
\end{tabular}

FUENTE: INE. 


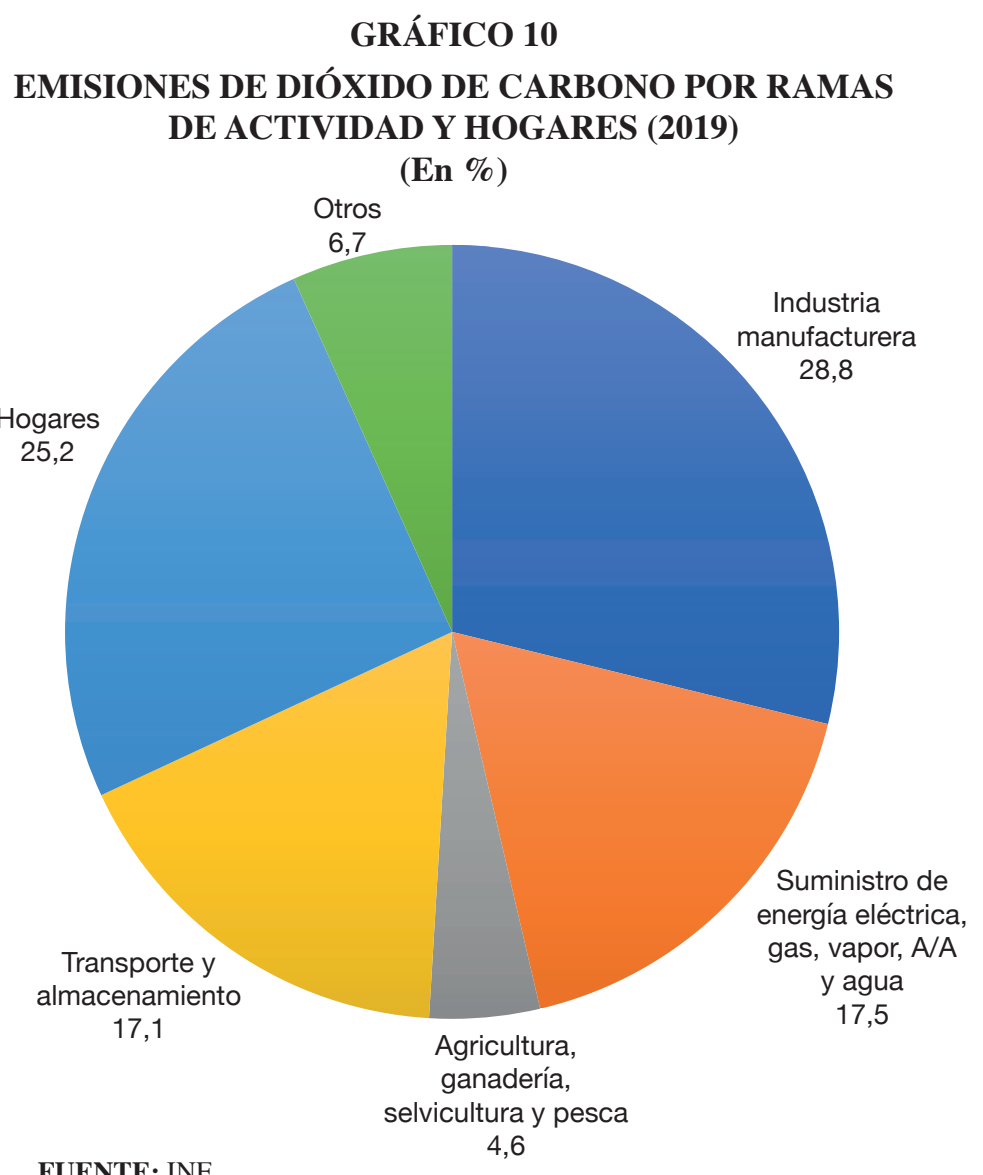

FUENTE: INE.

Por su parte, agricultura, ganadería, selvicultura y pesca emitió las mayores cantidades de metano y óxido nitroso. En concreto, el 63,6\% del total de dióxido de carbono equivalente de metano y el 78,0\% de óxido nitroso.

A partir de los datos recogidos en las Cuentas de emisiones a la atmósfera se pueden elaborar varios indicadores de desarrollo sostenible correspondientes a la Agenda 2030.

Para la consecución del objetivo 9.4, consistente en que para 2030 se debe modernizar la infraestructura y reconvertir las industrias para que sean sostenibles, utilizando los recursos con mayor eficacia, se obtiene el indicador 9.4.1, consistente en medir las emisiones de $\mathrm{CO}_{2}$ por unidad de valor añadido.

Para la consecución del objetivo 13.2, consistente en incorporar medidas relativas al cambio climático en las políticas, estrategias y planes nacionales, del cual a partir de las Cuentas de emisiones se puede obtener una aproximación para el indicador 13.2.1. Este indicador pretende medir el número de países que han comunicado el 
establecimiento o la puesta en funcionamiento de una estrategia/plan/política integrada que aumenta su capacidad para adaptarse a los efectos adversos del cambio climático y fomenta la resiliencia al cambio climático de bajas emisiones de gases efecto invernadero de una manera que no amenace la producción de comida, si bien al ser este un indicador de nivel III, se calcula un proxy siguiendo las recomendaciones de Naciones Unidas. El indicador usa como dato de total de emisiones de Gases de Efecto Invernadero (GEI) a la atmósfera el publicado por la Cuenta de Emisiones a la Atmósfera.

\section{Cuenta de los residuos}

\subsection{Marco conceptual}

Desde el año 1996, el INE elabora estadísticas sobre los residuos y, actualmente, lo hace conforme a las pautas establecidas en el Reglamento (CE) 2150/2002 del Parlamento Europeo y del Consejo, de 25 de noviembre, sobre estadísticas de residuos, modificado en virtud del Reglamento (UE) 849/2010.

Anualmente el INE elabora las Estadísticas de Generación, Recogida y Tratamiento de Residuos con el objetivo de cuantificar tanto el origen como el tratamiento de los mismos. Esta información, junto con la procedente de otros organismos, en especial la proporcionada por el Ministerio para la Transición Ecológica y Reto Demográfico, constituye la base para la elaboración de la Cuenta de los residuos.

La Cuenta de los residuos es útil para organizar la información sobre la generación de residuos y la gestión de sus flujos hacia instalaciones de reciclado, operaciones de relleno, incineración y vertederos controlados. A partir de ella se pueden obtener importantes indicadores para medir la presión que la economía ejerce sobre el medioambiente. También permite monitorizar los objetivos de las políticas de reducción de residuos y evaluar las mejoras en la gestión para la recuperación de materiales.

La estructura que a nivel europeo ha llevado al desarrollo normativo que ha resultado en la Cuenta de los residuos se asienta sobre los objetivos de la Política Europea de Residuos (PER), recogidos en la legislación europea, los cuales consistían en romper el vínculo entre el crecimiento económico y los impactos medioambientales asociados a la generación de residuos, mejorar la puesta en práctica de la legislación sobre los residuos, la prevención y gestión de los residuos (jerarquía de residuos), el movimiento hacia una «sociedad del reciclado» (este objetivo persigue eliminar la generación de residuos y usar los residuos como recursos) y, finalmente, impulsar la utilización de residuos para la producción de energía.

Sobre la base de estos objetivos, en 2013, se elaboró el $7^{\circ}$ Programa de acción ambiental para la Unión Europea, cuyos objetivos consistían en reducir la cantidad de residuos generados en los Estados miembros, maximizar el reciclaje y la reutilización, limitar la incineración a materiales no reciclables, eliminar gradualmente los 
vertidos de residuos no reciclables y no recuperables y garantizar la plena aplicación de los objetivos de la política de residuos en todos los Estados miembros ${ }^{6}$.

Para lograr varios de estos objetivos de la Política Europea de Residuos y posteriormente del $7^{\circ}$ Programa de acción ambiental, en 2008 se aprobó el marco jurídico para el tratamiento de los residuos en la Unión Europea, la Directiva Marco de Residuos , y en concreto, para lograr los objetivos de prevención y gestión de los residuos, y la utilización de residuos para la producción de energía, en la Directiva se incluyen normas concretas que afectan a la recogida separada de residuos, que buscan el impulso del reciclado mediante el establecimiento de objetivos y que establecen los criterios de «fin de residuo» o end of waste ${ }^{7}$.

Para cuantificar y poder comparar a nivel europeo los resultados de las políticas de residuos y el cumplimento de la Directiva, en 2002 se aprobó el Reglamento sobre Estadísticas de Residuos, entre cuyos objetivos se encuentran la armonización de datos de residuos en los Estados miembros a fin de que puedan compararse y agregarse, y proporcionar herramientas para las políticas públicas medioambientales, de desarrollo sostenible y de salud pública ${ }^{8}$. Este Reglamento establece una serie de elementos básicos a nivel estadístico para permitir la comparabilidad entre los datos publicados por los distintos Estados miembros, así como la definición de residuo, el uso de una nomenclatura estadística específica para las categorías de residuos, la Clasificación Estadística de Residuos (CER), la identificación de los flujos de generación de residuos estableciendo el origen de los mismos (en ámbito CNAE 2009 a partir de 2008), y la descripción de las operaciones de tratamiento (el destino final de los residuos).

Si bien la definición de residuo recogida en el Reglamento de residuos y en la Directiva de residuos es la misma en su literalidad ${ }^{9}$, a efectos del cómputo estadístico de los mismos existen diferencias, ya que conforme establece el Reglamento aquellos residuos que son reciclados internamente en las empresas o establecimientos que los generan o durante el proceso de tratamiento no se consideran como tales, sino como subproductos. Estas diferencias metodológicas conllevan discrepancias entre los datos recogidos conforme a una u otra norma.

\subsection{Metodología y estructura}

El Reglamento de residuos establece la diferencia entre la generación y el tratamiento. Los residuos generados pueden proceder de los procesos productivos, aunque no son un objetivo de los mismos. Aparecen principalmente en la industria, minería,

${ }^{6}$ Decision 1386/2013/EU of the European Parliament and of the Council of 20 November 2013 on a General Union Environment Action Programme to 2020 «Living well, within the limits of our planet».

7 Directiva 2008/98/CE del Parlamento Europeo y del Consejo del 19 de noviembre de 2008 sobre residuos (DMR).

${ }^{8}$ Reglamento (UE) 2150/2002 del Parlamento Europeo y del Consejo y de 25 de noviembre de 2002 sobre Estadísticas de Residuos modificado en 2010 por el Reglamento (UE) 849/2010 de la Comisión de 27 de septiembre de 2010.

9 Residuo: cualquier sustancia u objeto cuyo poseedor deseche o tenga la intención u obligación de desechar. 
agricultura, etc. Pueden considerarse fracciones de los flujos de salida de materiales y no son productos ni subproductos del proceso productivo. También hay generación de residuos en las actividades de consumo, principalmente del consumo privado, e incluyen los resultantes de la preparación de alimentos y de los trabajos domésticos. Pueden también generarse en empresas como, por ejemplo, las dedicadas a la hostelería. Y, finalmente, también se pueden generar en las instalaciones de tratamiento de residuos durante los propios procesos de tratamiento, que no producen bienes materiales, sino que cambian las características físicoquimicas de los residuos de entrada. Estos residuos generados deben ser tratados, y el Reglamento establece que solo deben considerarse las operaciones de tratamiento final. Estas operaciones vienen identificadas con los códigos D/R de la DMR, siendo D las operaciones de incineración sin recuperación de energía y vertido, y R las operaciones de recuperación (reciclado, compostaje, incineración con recuperación de energía, etc.).

A través de la metodología establecida en el Reglamento se recogen los datos de la generación de residuos en origen, incluyendo las cantidades generadas de cada categoría dentro de las fronteras del país por sectores de actividad y de los hogares como consumidores finales, descontando los residuos exportados. También se recogen los tratados en las operaciones de tratamiento final, recopilando los datos de las cantidades de residuos tratadas en el interior del país (tanto generadas en el país como importadas) de las distintas categorías, por operaciones de tratamiento y a nivel nacional.

Para poder compatibilizar los datos de generación con los de tratamiento se requiere información sobre exportaciones e importaciones, de tal forma que:

$$
(\text { Generación }- \text { Tratamiento })_{\text {Residuo i }}=(\text { Exportaciones }- \text { Importaciones })_{\text {Residuo i }}
$$

Estos residuos se recogen, tal y como establece el Reglamento, conforme a la Clasificación estadística de los residuos o clasificación CER ${ }^{10}$. Esta nomenclatura es específica para elaborar las estadísticas y Cuentas de residuos, está orientada a sustancias, incluye cuatro niveles de codificación o agregación y consta de 117 categorías a 4 dígitos, siendo excluyentes entre sí a igual nivel de agregación. Esta clasificación es diferente de la Lista Europea de Residuos (LER) que recoge la Directiva de residuos, que, con fines administrativos, constituye una relación de residuos armonizada a nivel europeo clasificados con hasta 6 dígitos, definiendo tanto el residuo como la fuente que lo genera, e identificando el status de peligrosidad de los mismos ${ }^{11}$. En el anexo III del Reglamento ${ }^{12}$ se incluye una tabla de equivalencias donde a cada categoría LER a 6 dígitos se le asigna una única categoría CER a 4 dígitos, de tal forma que para poder calcular el total de una categoría CER se agregan las cantidades de residuos de los distintos códigos LER que la componen.

\footnotetext{
${ }^{10}$ Cer-Stat Ver.4 - https://www.boe.es/buscar/doc.php?id=DOUE-L-2010-81762

11 Establecida por la Decisión 2000/532/CE de la Comisión.

12 https://www.ine.es/daco/daco42/resiurba/reglamento.pdf
} 
La Cuenta de los residuos recoge los datos del flujo de estos mediante la elaboración de dos matrices, una de generación y otra de tratamiento. Las filas de las matrices recogen las cantidades respectivas de residuos generados repartidas en las 51 categorías establecidas en la clasificación CER, siendo 30 categorías de residuos no peligrosos y el resto de peligrosos ${ }^{13}$. Las columnas de las matrices diferencian entre el origen o generación de los residuos y el destino o tratamiento de los mismos. El origen incluye todas las actividades económicas agrupadas en 20 rúbricas de la Clasificación Nacional de Actividades Económicas (CNAE 2009 ${ }^{14}$ ) y también incluye a los hogares como consumidores finales y los residuos generados en las operaciones de recuperación o eliminación. El destino solo tiene en cuenta las operaciones de tratamiento final agrupadas en 6 bloques (recuperación de energía, incineración, valorización, backfling, vertido, otros vertidos).

\subsection{Elaboración}

Para poder elaborar la Cuenta de residuos, es necesario obtener información completa y precisa tanto de la generación como de los procesos de tratamiento. Esta información se obtiene mediante encuestas, mediante fuentes administrativas o fuentes relacionadas con obligaciones de información bajo legislación comunitaria relativa a la gestión de residuos, mediante la información recogida en el Registro Estatal de Emisiones y Fuentes Contaminantes (PRTR) ${ }^{15}$, y mediante modelos de estimación basados en la vinculación de los residuos con variables auxiliares. Cada una de estas fuentes de información tiene asociados una serie de ventajas e inconvenientes. Así, las fuentes administrativas presentan un coste cero en la obtención de la información, pero requieren de más laboriosidad en cuanto a la adaptación de los datos a los requisitos del Reglamento de residuos. La estimación basada en modelos es apropiada cuando se dispone de variables auxiliares claramente relacionadas con los residuos, pero la elaboración de los modelos no es sencilla y puede conllevar a errores de estimación. Y en cuanto a las encuestas presentan la ventaja de la inmediatez, ya que se realizan con el objetivo de cumplir con los requisitos establecidos en el Reglamento de residuos, pero a cambio presentan el coste económico más elevado.

Los datos de la generación de residuos de las actividades económicas y de los hogares como consumidores finales se obtienen de diversas fuentes. Por un lado, se dispone de información procedente de cinco operaciones estadísticas elaboradas por el INE, a saber, la encuesta de generación de residuos en la agricultura y ganadería

13 «Residuo peligroso»: un residuo se considera peligroso cuando presenta unas determinadas características de peligrosidad y, por tanto, es necesario someterlo a exigencias adicionales de control para evitar que pueda provocar daños a la salud o al medio ambiente, durante su producción y gestión. Más información acerca de cómo se clasifican los residuos como peligrosos en https:/www.miteco.gob.es/es/calidad-y-evalau cion-ambiental/temas/prevencion-y-gestion-residuos/flujos/Residuos_con_caracteristicas_peligrosidad.aspx

14 https://www.cnae.com.es/

15 http://www.prtr-es.es/ 
y el censo agrario para los residuos procedentes de la agricultura y la ganadería, la encuesta de generación de residuos en la pesca y la encuesta de generación de residuos en la acuicultura para los sectores de la pesca y la acuicultura; la encuesta de generación de residuos en los servicios y construcción para los residuos procedente de las actividades de construcción y de las actividades de servicios; y finalmente, la encuesta de generación de residuos en la industria para los que proceden de las actividades industriales. También se obtiene información a partir de la encuesta de recogida y tratamiento de residuos que recoge datos sobre esas actividades y también sobre la generación de los hogares procedentes de los datos de las recogidas municipales. Por otro lado, se dispone de información sobre generación de residuos procedente de registros administrativos, en concreto del Registro Estatal de Emisiones y Fuentes Contaminantes (PRTR). En combinación con modelos de estimación, esa información proporciona datos de algunas ramas de actividad y sirve de base para estimar las cantidades generadas en aquellos años en que no hay disponibilidad de datos de encuestas.

Los datos del tratamiento de los residuos se obtienen, fundamentalmente, de la encuesta de recogida y tratamiento de residuos que anualmente elabora el INE. Además, se utilizan otras fuentes de información como datos proporcionados por MITERD, datos de importaciones y exportaciones de residuos, etc.

El objetivo de la medición del tratamiento de residuos es cuantificar en unidades físicas los flujos de materiales asociados con las actividades de gestión de residuos en el interior del país, para cada categoría de residuos y por tipo de operación de tratamiento. Estos datos se obtienen de la información proporcionada por las empresas encargadas de los servicios de recogida y transporte de residuos, y de la gestión de centros de transferencia y de tratamiento (reciclado o eliminación) de los mismos.

Dentro de las actividades de tratamiento de residuos se incluyen la recogida por medio de depósitos y envases adecuados, la recogida selectiva de residuos de los centros y emplazamientos especiales y el tratamiento y eliminación de residuos sólidos, por incineración u otros medios de reducción, mediante el vertido o por técnicas de inertización.

\subsection{Estimaciones e indicadores}

A continuación se presentan algunos de los resultados obtenidos a partir de la Cuenta de residuos (Cuadro 7).

La economía española generó 137,8 millones de residuos en 2018. De estos, 3,2 millones correspondieron a residuos peligrosos y 134,6 , millones a residuos no peligrosos.

Como recoge el Cuadro 7, las categorías de residuos generados más relevantes fueron residuos minerales (72,3 millones de toneladas), residuos mezclados $(37,8$ millones de toneladas) y residuos de animales y vegetales (9,3 millones de toneladas). El 97,6 \% de los residuos generados en 2018 fueron residuos no peligrosos y 


\section{CUADRO 7}

RESIDUOS GENERADOS POR TIPO DE RESIDUO* (2018)

(En miles de toneladas)

\begin{tabular}{|c|c|c|c|c|c|c|}
\hline & Total & $\%$ & $\begin{array}{c}\text { No } \\
\text { peligrosos }\end{array}$ & $\%$ & Peligrosos & $\%$ \\
\hline Residuos generados & $137.822,9$ & 100,0 & $128.884,6$ & 100,0 & $3.224,2$ & 100,0 \\
\hline Minerales & $72.341,3$ & 52,5 & $71.756,9$ & 53,4 & 584,4 & 18,1 \\
\hline Residuos mezclados & $37.849,2$ & 27,5 & $37.765,2$ & 28,1 & 84,0 & 2,6 \\
\hline Animales y vegetales & $9.291,7$ & 6,7 & $9.291,7$ & 6,9 & .. & .. \\
\hline Metálicos & $5.977,3$ & 4,3 & $5.977,3$ & 4,4 & .. & .. \\
\hline Papel y Cartón & $3.379,3$ & 2,5 & $3.379,3$ & 2,5 & .. & .. \\
\hline Químicos & $2.325,0$ & 1,7 & 981,4 & 0,7 & $1.343,6$ & 41,7 \\
\hline Lodos comunes & $1.603,3$ & 1,2 & $1.603,3$ & 1,2 & .. & .. \\
\hline Equipos desechados & $1.483,0$ & 1,1 & 305,7 & 0,2 & $1.177,3$ & 36,5 \\
\hline Vidrio & $1.170,4$ & 0,8 & $1.170,4$ & 0,9 & 0,0 & 0,0 \\
\hline Madera & $1.115,9$ & 0,8 & $1.114,9$ & 0,8 & 1,0 & 0,0 \\
\hline Plásticos & 815,3 & 0,6 & 815,3 & 0,6 & .. & .. \\
\hline Otros & 471,2 & 0,3 & 437,3 & 0,3 & 33,9 & 1,1 \\
\hline
\end{tabular}

NOTA: * El símbolo .. indica las categorías de residuos no contempladas por el Reglamento CE 2150/2002. FUENTE: INE.

el 2,4\% residuos peligrosos. Del total de los peligrosos, el 41,7\% correspondieron a residuos químicos, el $36,5 \%$ a equipos desechados y el 18,1\% a residuos minerales. Por tipo de residuos, los generados por los hogares con mayor peso fueron los residuos mezclados $(83,8 \%$ del total), papel y cartón $(4,7 \%)$ y animales y vegetales $(4,4 \%)$.

El Cuadro 8 recoge los residuos generados por los sectores de actividad. Como puede observarse los principales fueron los residuos minerales $(62,4 \%$ del total) y los residuos mezclados $(16,4 \%)$.

Por sectores, los residuos minerales fueron originados principalmente por la construcción (37,6 millones de toneladas) y la industria (32,4 millones de toneladas). Y los residuos mezclados por el suministro de agua, saneamiento, gestión de residuos y descontaminación (16,4 millones de toneladas).

Por su parte, los residuos animales y vegetales, fueron generados en su mayor parte por la agricultura, ganadería, silvicultura y pesca (5,7 millones de toneladas) (Gráfico 11).

Por sectores de actividad, el que más residuos generó fue la industria, el 29,5\% del total de los residuos, seguida por la construcción con el 27,6\% y por el suministro de agua, saneamiento, gestión de residuos y descontaminación, con el 17,6\% del total de los residuos generados. 


\section{CUADRO 8 \\ TIPOS DE RESIDUOS GENERADOS POR SECTORES DE ACTIVIDAD \\ Y HOGARES (2018) \\ (En miles de toneladas)}

\begin{tabular}{|l|r|r|r|r|r|r|}
\hline & \multicolumn{1}{|c|}{ Total } & $\boldsymbol{\%}$ & $\begin{array}{r}\text { Sectores de } \\
\text { actividad }\end{array}$ & \% & Hogares & \% \\
\hline Residuos generados & $137.822,9$ & 100,0 & $115.123,7$ & 100,0 & $22.699,2$ & 100,0 \\
\hline Minerales & $72.341,3$ & 52,5 & $71.868,8$ & 62,4 & 472,5 & 2,1 \\
\hline Residuos mezclados & $37.849,2$ & 27,5 & $18.833,0$ & 16,4 & $19.016,2$ & 83,8 \\
\hline Animales y vegetales & $9.291,7$ & 6,7 & $8.281,7$ & 7,2 & $1.010,0$ & 4,4 \\
\hline Metálicos & $5.977,3$ & 4,3 & $5.949,4$ & 5,2 & 27,9 & 0,1 \\
\hline Papel y Cartón & $3.379,3$ & 2,5 & $2.311,9$ & 2,0 & $1.067,4$ & 4,7 \\
\hline Químicos & $2.325,0$ & 1,7 & $2.315,7$ & 2,0 & 9,3 & 0,0 \\
\hline Lodos comunes & $1.603,3$ & 1,2 & $1.603,3$ & 1,4 &.. &.. \\
\hline Equipos desechados & $1.483,0$ & 1,1 & $1.422,4$ & 1,2 & 60,6 & 0,3 \\
\hline Vidrio & $1.170,4$ & 0,8 & 335,7 & 0,3 & 834,7 & 3,7 \\
\hline Madera & $1.115,9$ & 0,8 & 979,7 & 0,8 & 136,2 & 0,6 \\
\hline Plásticos & 815,3 & 0,6 & 791,2 & 0,7 & 24,1 & 0,1 \\
\hline Otros & 471,2 & 0,3 & 430,9 & 0,4 & 40,3 & 0,2 \\
\hline
\end{tabular}

NOTA: * El símbolo .. indica las categorías de residuos no contempladas por el Reglamento (CE) 2150/2002. FUENTE: INE.

\section{GRÁFICO 11}

\section{RESIDUOS GENERADOS POR SECTORES DE ACTIVIDAD}

Y HOGARES (2018)

(En \%)

Agricultura, ganadería, silvicultura y pesca, 4,5

Hogares, 16,5

Servicios, 4,3

Industria (incluidas minería y producción de energía), 29,5 
De los 137,8 millones de toneladas de residuos generados en 2018, las empresas de tratamiento final de residuos ${ }^{16}$ gestionaron 122,0 millones de toneladas de residuos.

En concreto, trataron 119,6 millones de toneladas de residuos no peligrosos y 2,3 millones de residuos peligrosos.

Del total de residuos tratados, el 48,2\% acabaron en el vertedero, el 38,7\% se reciclaron, el 10,0\% se reutilizaron en operaciones de relleno y el 3,1\% se incineraron (Gráfico 12).

\section{GRÁFICO 12 \\ TRATAMIENTO FINAL DE RESIDUOS (2018) \\ (En \%)}

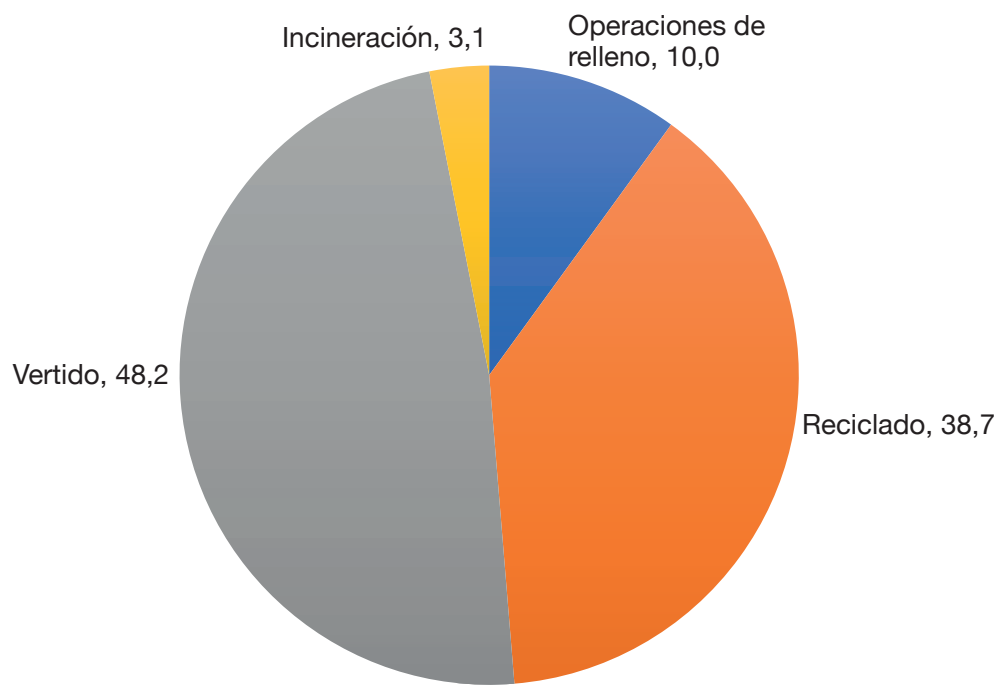

FUENTE: INE

En 2018 se reciclaron 47,2 millones de toneladas de residuos de las que el 44,8\% correspondió a residuos minerales, el 22,7 \% a los metálicos y el 8,3 \% a papel y cartón.

A partir de los datos recogidos en la Cuenta de los residuos se pueden elaborar varios indicadores de desarrollo sostenible correspondientes a la Agenda 2030.

Para la consecución del objetivo 11.6, consistente en que para 2030 se debe reducir el impacto ambiental negativo per cápita de las ciudades, incluso prestando especial atención a la calidad del aire y la gestión de los desechos municipales y de otro tipo, se obtiene el indicador 11.6.1, consistente en la proporción de desechos sólidos urbanos recogidos periódicamente y con una descarga final adecuada respecto del total de desechos sólidos urbanos generados, desglosada por ciudad.

${ }^{16}$ Solo se incluyen tratamientos finales. Las principales diferencias entre los residuos generados y tratados publicados en la Cuenta de los residuos se deben, entre otros motivos, a contabilizar exclusivamente los tratamientos finales, al comercio exterior de residuos (importaciones y exportaciones) y a las diferencias de volumen entre el momento en que los residuos son generados y finalmente tratados. 
Para la consecución del objetivo 12.4, consistente en lograr la gestión ecológicamente racional de los productos químicos y de todos los desechos a lo largo de su ciclo de vida, de conformidad con los marcos internacionales convenidos, y reducir significativamente su liberación a la atmósfera, el agua y el suelo a fin de minimizar sus efectos adversos en la salud humana y el medioambiente, se obtiene de los datos de la Cuenta de los residuos el indicador 12.4.2, consistente en la cantidad de desechos peligrosos generados per cápita y en la proporción de desechos peligrosos tratados, desglosados por tipo de tratamiento.

\section{A modo de conclusión: perspectivas futuras de las cuentas físicas medioambientales}

A la vista de los desafíos medioambientales que tienen planteados el planeta Tierra en cuanto a la sostenibilidad del desarrollo económico y social de sus habitantes, las cuentas ambientales físicas van a jugar en los años venideros un importante papel para calibrar y cuantificar la viabilidad de los modelos de desarrollo económico. por su impacto en las variaciones tanto en cantidad como en calidad, del stock de activos/ recursos naturales/ecosistemas definido dicho stock como el inventario de activos/recursos medioambientales renovables y no renovables (minerales, agua, aire, suelo, biodiversidad).

La recogida de datos medioambientales presenta muchas dificultades, no siendo la menor de ellas la ausencia de una «contabilidad física medioambiental» armonizada normativamente y de aplicación a las actividades económicas, especialmente a las que se refieren a los procesos industriales. Otra cuestión ciertamente relevante en el campo de las cuentas físicas medioambientales atañe a los problemas derivados de la multiplicidad de unidades de medida físicas, lo que conlleva la necesidad de reconvertirlas a una sola unidad con los consiguientes sesgos originados por las equivalencias de medidas.

Las perspectivas futuras de este tipo de cuentas pasan, en lo que respecta a las de materiales físicos y de residuos, por integrarse en el marco conceptual de lo que ha venido a denominarse la «economía circular». Para ello es necesario investigar otros aspectos de los procesos industriales como por ejemplo el empleo como input en dichos procesos de materiales reciclados. En lo que respecta a la Cuenta de emisiones a la atmósfera y a la de flujos físicos de energía, los trabajos internacionales a corto y medio plazo procuran establecer una mayor coordinación entre ambas cuentas de tal forma que queden identificados los productos energéticos cuyo uso genera más emisiones a la atmósfera.

Carácter complementario pero muy relevante tiene la Cuenta del capital natural cuyo objetivo es valorar económicamente la naturaleza, asignando un valor monetario a todos los servicios que presta, y a partir de ahí, compensar económicamente el impacto que las actividades humanas tienen sobre los ecosistemas. Esta cuenta, a caballo entre lo físico y lo monetario, tiene sus defensores y detractores, argumentando 
estos últimos la gran dificultad que supone valorar en términos monetarios activos y servicios que no son de mercado. En cualquier caso, con esta cuenta el impacto se integraría en los cálculos de costes/beneficios de las actividades económicas.

En este contexto, cabe señalar que los intentos metodológicos para desarrollar lo que ha venido en denominarse el «PIB verde», es decir la estimación del crecimiento o disminución del PIB monetario teniendo en cuenta la depreciación de los activos naturales, han puesto en evidencia los problemas que se plantean cuando se intenta valorar en términos monetarios los recursos naturales. Por ello es preciso avanzar en la integración de las cuentas ambientales físicas con las monetarias, todo ello en sintonía con el marco conceptual del SEC.

\section{Referencias bibliográficas}

Brundtland, G. H. (1987). Informe de la Comisión Mundial sobre el Medio Ambiente y el Desarrollo: Nuestro futuro común. Naciones Unidas. https://undocs.org/es/A/42/427

Celestino Rey, F., Solera Carnicero, A. L. \& Martínez Serrano, A. (2018). Las cuentas medioambientales y la cuenta de los flujos físicos de la energía. Economía Industrial, 408, 143-152.

Decisión 1386/2013/EU del Parlamento europeo y del Consejo, de 20 de noviembre de 2013, relativa al Programa General de Acción de la Unión en materia de Medio Ambiente hasta 2020 «Vivir bien, respetando los límites de nuestro planeta». Diario Oficial de la Unión Europea. https://eur-lex.europa.eu/legal-content/ES/TXT/PDF/?uri=CELEX: 32013D1386\&from=ES

Directiva 2008/98/CE del Parlamento Europeo y del Consejo del 19 de noviembre de 2008 sobre residuos y por la que se derogan determinadas Directivas. Diario Oficial de la Unión Europea. https://eur-lex.europa.eu/legal-content/ES/TXT/PDF/?uri=CELEX: 32008L 0098\&from $=\mathrm{ES}$

European Commission (2020). Combined Nomenclature for external trade statistics. https://ec.europa.eu/taxation_customs/business/calculation-customs-duties/what-iscommon-customs-tariff/combined-nomenclature_en

Eurostat (2014). Physical Energy Flows Accounts (PEFA)._https://ec.europa.eu/eurostat/ documents/1798247/6191537/PEFA-Manual-2014-v20140515.pdf

Eurostat (2015). Manual for air emissions accounts. https://ec.europa.eu/eurostat/web/ products-manuals-and-guidelines/-/KS-GQ-15-009

Eurostat (2018). Economy-wide material flow accounts. Handbook. https://ec.europa.eu/ eurostat/documents/3859598/9117556/KS-GQ-18-006-EN-N.pdf/b621b8ce-2792-47ff$9 \mathrm{~d} 10-067 \mathrm{~d} 2 \mathrm{~b} 8 \mathrm{aac} 4 \mathrm{~b}$

Eurostat (2020). Material flows in the circular economy. https://ec.europa.eu/eurostat/ statistics-explained/index.php?title=Material_flows_in_the_circular_economy

INE, Instituto Nacional de Estadística. (2002). Balance y cuenta de flujos de materiales. https://ine.es/daco/daco42/ambiente/aguasatelite/metflujos1.pdf

INE, Instituto Nacional de Estadística. (2020, 16 de diciembre). Cuentas medioambientales: Cuenta de flujos de materiales. Datos avance 2019. https://www.ine.es/prensa/cma_2019_fm.pdf 
Naciones Unidas, Comisión Europea, Organización de Cooperación y Desarrollo Económicos, Fondo Monetario Internacional \& Grupo del Banco Mundial. (2008). Sistema de Cuentas Nacionales.

https://unstats.un.org/unsd/nationalaccount/docs/SNA2008Spanish.pdf

ONU, Organización de las Naciones Unidas. (2016). Sistema de contabilidad ambiental y económica 2012. Marco Central. https://unstats.un.org/unsd/envaccounting/seearev/CF_trans/SEEA_CF_Final_sp.pdf

Reglamento (CE) n 2150/2002 del Parlamento europeo y del Consejo, relativo a las estadísticas sobre residuos. Diario Oficial de las Comunidades Europeas, n. ${ }^{\circ} 332$, de 9 de diciembre de 2002. https://www.boe.es/buscar/doc.php?id=DOUE-L-2002-82232

Reglamento (UE) n. ${ }^{\circ}$ 691/2011 del Parlamento europeo y del Consejo, relativo a las cuentas económicas europeas medioambientales. Diario Oficial de la Unión Europea. https://eur-lex.europa.eu/legal-content/ES/TXT/PDF/?uri=CELEX:32011R0691\&from=EN

Reglamento (UE) n ${ }^{\circ}$ 538/2014 del Parlamento Europeo y del Consejo de 16 de abril de 2014 por el que se modifica el Reglamento (UE) No 691/2011, relativo a las cuentas económicas europeas medioambientales. Diario oficial de la Unión Europea. https://www.boe.es/doue/2014/158/L00113-00124.pdf

Sanyé-Mengual, E., Secchi, M., Corrado, S., Beylot, A., \& Sala, S. (2019). Assessing the decoupling of economic growth from environmental impacts in the European Union: A consumption-based approach. Journal of cleaner production, 236, 117535. https:/www.sciencedirect.com/science/article/pii/S0959652619323431?via\%3Dihub

UN, United Nations. (2018). International Recommendations for Energy Statistics. https://unstats.un.org/unsd/energy/ires/IRES-web.pdf

UNEP, United Nations Environment Programme's. (2020). Sustainable Trade in Resources: Global Material Flows, Circularity and Trade.

https://www.resourcepanel.org/reports/sustainable-trade-resources 